GABRIEL TORTELLA DE SOUZA

Segurança cognitiva no tratamento da depressão bipolar com estimulação transcraniana por corrente contínua: um ensaio clínico randomizado, duplo-cego, placebo-controlado

São Paulo

2019 
GABRIEL TORTELLA DE SOUZA

\title{
Segurança cognitiva no tratamento da depressão bipolar com estimulação transcraniana por corrente contínua: um ensaio clínico randomizado, duplo-cego, placebo-controlado
}

\author{
Tese apresentada à Faculdade de Medicina da \\ Universidade de São Paulo para obtenção de título \\ de Doutor em Ciências \\ Programa de Ciências Médicas \\ Área de concentração: Educação e Saúde \\ Orientador: Prof. Dr. Andre Russowsky Brunoni
}

(Versão corrigida. Resolução CoPGr 6018/11, de 13 de outubro de 2011. A versão original está disponível na Biblioteca da FMUSP)

\section{São Paulo}


Dados Internacionais de Catalogação na Publicação (CIP)

Preparada pela Biblioteca da

Faculdade de Medicina da Universidade de São Paulo

C)reprodução autorizada pelo autor

Souza, Gabriel Tortella de

Segurança cognitiva no tratamento da depressão bipolar com estimulação transcraniana por corrente contínua : um ensaio clínico randomizado, duplo-cego, placebo-controlado / Gabriel Tortella de Souza. -São Paulo, 2019.

Tese (doutorado)--Faculdade de Medicina da Universidade de são Paulo.

Programa de Ciências Médicas.Área de Concentração: Educação e Saúde.

Orientador: Andre Russowsky Brunoni.

Descritores: 1.Transtorno bipolar 2.Depressão 3. Estimulação transcraniana por corrente contínua 4. Cognição 5. Avaliação cognitiva

USP / FM/DBD-130/19

Responsável: Erinalva da Conceição Batista, CRB-8 6755

\section{Dedicatória}


Para minha esposa Jéssica e minha família, Carlos, Rosana e Rafael. 


\section{Agradecimentos}

A Deus pela oportunidade da vida.

A todos os participantes que se voluntariaram a projeto, sem os quais a realização desta pesquisa não seria possível.

Aos profissionais do CINA HU envolvidos de forma responsável neste trabalho.

Ao Prof. Dr. Andre Russowski Brunoni, que me orientou de forma paciente e comprometida ao longo da minha caminhada na pós-graduação. 
Página

$\begin{array}{ll}\text { Lista de siglas } & 7\end{array}$

$\begin{array}{lr}\text { Lista de figuras e tabelas } & 8\end{array}$

$\begin{array}{ll}\text { RESUMO } & 8\end{array}$

$\begin{array}{ll}\text { ABSTRACT } & 10\end{array}$

1. INTRODUÇÃO 12

1.1 Transtorno de Humor Bipolar 12

1.2 Fundamentação Teórica 15

1.3 Estimulação Transcraniana por Corrente Contínua $\quad 20$

1.4 Ensaio de Tratamento Elétrico de Depressão Bipolar 22 [BETTER]

2. MATERIAL E MÉTODOS 24

2.1 Objetivos 24

2.2 Hipóteses 25

2.3 Desenho do estudo $\quad 26$

2.4. ANÁLISE ESTATÍSTICA 36

3. RESULTADOS 38

4. DISCUSSÕES 48

5. CONCLUSÕES 50

6. BIBLIOGRAFIA 51

Anexo 


\section{LISTA DE SIGLAS}

BETTER Bipolar Depression Electrical Treatment Trial

TB

Transtorno Bipolar

HDRS17

Escala de Depressão de Hamilton - 17 itens

ETCC

Estimulação transcraniana por corrente contínua

YRMS

Escala de Avaliação de Mania de Young

DLPFC

Cortical pré-frontal dorsolateral

MINI

Mini International Neuropsychiatric Interview

MADRS

Montgomery-Åsberg Depression Rating Scale

CGI

Escala de Impressão Clínica Global

BDI

Inventário de Depressão de Beck

RAVLT

Teste de Aprendizagem Auditivo-Verbal de Rey 


\section{LISTA DE TABELAS E FIGURAS}

Página

Tabela 1 Fluxograma para cada paciente, ao longo do estudo. 26

Figura 1 Desligamento automático do equipamento simulado após trinta 30

Tabela 2 Bateria neuropsicológica. 36

Figura 2 Fluxograma dos pacientes encaminhados e incluídos no estudo. 38

Tabela 3 Características clínicas e demográficos da amostra no início do 39 estudo.

Tabela 4 Escores dos testes neuropsicológicos ao longo do tempo em grupos 40

Tabela $5 \quad$ Características cognitivas da amostra no início e final do estudo. $\quad 41$

Tabela 6 Diferença entre o grupo placebo e o ativo no início e no final do 43 estudo.

Resultados do modelo misto para os efeitos principais e de 44

Tabela 7 interação dos fatores tempo, grupo, tempo x grupo e depressão.

Alterações cognitivas ao longo do tempo em grupos ativos vs. 45

Figura 3 simulados.

Correlação de Pearson entre mudanças em 5 domínios cognitivos:

Figura 4 memória; memória de trabalho; atenção; língua; controle e placebo (lado superior direito). 


\section{RESUMO}

Souza GT. Segurança cognitiva no tratamento da depressão bipolar com estimulação transcraniana por corrente contínua: um ensaio clínico randomizado, duplo-cego, placebocontrolado [Tese]. São Paulo: Faculdade de Medicina da Universidade de São Paulo; 2019.

A depressão bipolar é um quadro psiquiátrico que tem entre seus sintomas o comprometimento das habilidades cognitivas. Os tratamentos farmacológicos usados para essa condição podem agravar os déficits cognitivos do paciente. Nesse contexto, surge a necessidade de uma opção terapêutica que preserve a cognição. O objetivo deste estudo foi investigar se a Estimulação Transcraniana por Corrente Contínua (ETCC) é uma ferramenta terapêutica que apresenta segurança cognitiva no tratamento de pacientes com depressão bipolar. Foi realizado um estudo randomizado, controlado por placebo, duplo-cego (Bipolar Depression Electrical Treatment Trial [BETTER]). Cinquenta e nove pacientes (40\% mulheres), com transtorno bipolar em um episódio depressivo maior (pontuação superior a 17 para a Escala de Depressão de Hamilton - HDRS17) foram randomizados em dois grupos. Eles foram seguidos por uma bateria de avaliações neuropsicológicas, antes do tratamento, após duas e seis semanas. A ETCC foi aplicada diariamente por dez dias consecutivos, exceto aos finais de semana, e depois 1 sessão a cada quinzena até a semana 6 , na região do córtex pré-frontal dorsolateral, por de 30 minutos (2-mA, ânodo a esquerdo e cátodo a direita). Não houve diferença significativa entre o grupo placebo e o grupo tratado com ETCC no início e no final do estudo, para as características cognitivas. Não foi encontrado comprometimento cognitivo para pacientes tratados com ETCC. Este é um achado importante quando 
comparado a técnicas farmacológicas que podem apresentar efeitos colaterais cognitivos durante o tratamento. Estudos adicionais devem examinar a segurança cognitiva da técnica com amostras maiores.

Descritores: Transtorno afetivo bipolar, depressão, Estimulação transcraniana por corrente contínua, cognição e avaliação cognitiva. 


\section{ABSTRACT}

Souza GT. Cognitive safety in the treatment of bipolar depression with transcranial direct current stimulation: a randomized, double-blind, placebo-controlled trial [Thesis]. São Paulo: Faculty of Medicine, University of São Paulo; 2019.

Bipolar depression is a psychiatric condition that has among its symptoms the impairment of cognitive abilities. The pharmacological treatments used for this condition may aggravate the patient's cognitive deficits. In this context, the need for a therapeutic option that preserves cognition arises. The aim of this study was to investigate if Transcranial Direct Current Stimulation (tDCS) is a therapeutic tool that presents cognitive safety in the treatment of patients with bipolar depression. A randomized, placebo-controlled, double-blind study (Bipolar Depression Electrical Treatment Trial [BETTER]) was performed. Fifty-nine patients (40\% women) with bipolar disorder in a major depressive episode (score above 17 for the Hamilton Depression Scale - HDRS17) were randomized into two groups. They were followed by a battery of neuropsychological assessments, before treatment, after two and six weeks. The ETCC was applied daily for 10 consecutive days, except on weekends, and then 1 session every fortnight until week 6 , in the region of the dorsolateral prefrontal cortex, for 30 minutes (2-mA, anode to left and cathode on the right). There was no significant difference between the placebo group and tDCS at the beginning and end of the study for all cognitive characteristics. No cognitive impairment was found for patients treated with tDCS. This is an important finding when compared to pharmacological techniques that may present cognitive side effects during treatment. Additional studies should examine the cognitive safety of the technique with larger samples. 
Descriptors: Bipolar affective disorder, depression, transcranial direct current stimulation, cognition and cognitive evaluation. 


\section{INTRODUÇÃO}

\subsection{Transtorno de Humor Bipolar}

O Transtorno Bipolar (TB), originalmente chamado de "insanidade maníacodepressiva", é uma doença caracterizada pela presença de episódios com sintomas clinicamente significativos de hipomania ou mania freqüentemente intercalados por períodos de normalidade do humor e de depressão. A prevalência estimada ao longo da vida é de aproximadamente 0,9 a 2,1\% (Merikangas et al., 2011). O TB inicia-se, em geral (até 60\% dos casos), no final da adolescência ou no início da vida adulta. É recorrente, com alterações de humor frequentemente presentes durante diversos momentos ao longo da vida, sendo as depressões mais prevalentes do que a euforia, ocorrendo em até $1 / 3$ do tempo da vida destes pacientes. As taxas de morbi-mortalidade são altas, incluindo risco de suicídio que chega a 15\% (Geddes \& Miklowitz, 2013; Yatham et al., 2013).

Os sintomas afetivos, cognitivos e neurovegetativos do TB estão associados a alterações anatômicas, neuroquímicas ou metabólicas no corpo estriado, tálamo, córtex préfrontal, estruturas límbicas (amígdala e o hipocampo) e cerebelo, entre outras. A hiperfunção de áreas límbicas e subcorticais (estriado, tálamo, amígdala) e a redução de modulação das áreas pré-frontais, seriam responsáveis pela desregulação das emoções e da cognição. Encontram-se alterações celulares, como redução de número e volume de neurônios e de células da glia no córtex pré-frontal dorsolateral, no giro do cíngulo anterior, no hipocampo e na amígdala. Tais alterações celulares podem eventualmente representar perda e atrofia celular ao longo do curso da doença (Gigante et al., 2011). As alterações de neurotransmissores (na mania observou-se hiperatividade adrenérgica e dopaminérgica e na 
depressão diminuição de atividade serotoninérgica) são consequências de disfunções complexas da transdução intracelular de sinais e da expressão gênica. Além disso, foram também encontradas alterações nos sistemas de neurotransmissão gabaérgico, glutamatérgico e de neuropeptídeos (Geddes \& Miklowitz, 2013; Phillips \& Kupfer, 2013).

Pacientes com TB apresentam prejuízos no desempenho dos domínios cognitivos, mesmo quando eutímicos. Revisões de literatura apontam que as principais habilidades com comprometimento cognitivo no TB são a atenção, memória de trabalho, funções executivas, memória episódica, memória verbal e velocidade de processamento de informação (Millan et al., 2012; Lima et al., 2019). Tais disfunções executivas podem, em parte, explicar as dificuldades na adaptação psicossocial dos pacientes com TB. Durante as alterações de humor, mesmo que discretos, são comumente notados a presença de déficits cognitivos (Depp et al., 2012).

O DSM- 5 continua dividindo os transtornos bipolares em: tipo I, caracterizado pela presença de episódio maníaco, dando ênfase à necessidade da presença do aumento de atividade ou energia; tipo II, caracterizado pela alternância de episódios hipomaníacos e depressivos (esta não é, no entanto, entendida como forma mais atenuada da doença, uma vez que indivíduos passam muito tempo em depressão e tem repercussões importantes com a constante instabilidade de humor); transtorno ciclotímico, no qual sintomas de elevação de humor se alternam com sintomas depressivos por pelo menos 2 anos (e para crianças o mínimo de 1 ano) sem, contudo, preencher critérios para episódio maníaco, hipomaníaco ou depressão maior. Como mudança ao DSM-IV-TR, versão anterior, episódios mistos (explicados em mais detalhes adiante) não são mais uma categoria independente, mas podem estar presentes durante episódios de mania/hipomania ou depressão maior. Outros 
especificadores adicionados foram "com ansiedade" (pelo menos 2 dos seguintes sintomas: tensão, inquietação, problemas de concentração pela preocupação, medo que algo horrível possa acontecer, sensação de perda de controle de si mesmo) e "risco suicida".

O suicídio é a principal causa de mortalidade precoce nestes pacientes. Os pacientes com TB apresentam risco 28 vezes maior de comportamento suicida em relação à população geral. A relação entre tentativa de suicídio e suicídio completo no TB é 5:1 contra 15:1 na população geral, indicando que esses pacientes tendem a usar métodos mais violentos e letais. As relações entre comportamento suicida e TB parecem ser também mais marcantes do que em outros transtornos psiquiátricos, sendo a mais importante entre homens e em segundo lugar entre mulheres. De $20 \%$ a $55 \%$ dos pacientes com TB já apresentou ao menos uma tentativa de suicídio ao longo da vida e as taxas de suicídios completos estão entre $10 \%$ a 15\% (Goodwin e Jamison, 2007; Yatham et al., 2013).

O objetivo do tratamento do TB é alcançar a eutimia, promover a prevenção de novos episódios de alteração de humor, além da recuperação do funcionamento social e ocupacional do indivíduo. Como normalmente é um tratamento por toda vida, deve-se atentar aos seus efeitos de longo prazo e seus possíveis prejuízos para a saúde. O TB ainda é uma condição psiquiátrica de difícil tratamento, em especial em relação a depressão bipolar, os estados mistos e os quadros de ciclagem rápida. Opções terapêuticas para depressão podem piorar as fases com sintomas maníacos e medicamentos para mania podem trazer uma depressão posterior. O conhecimento limitado da etiologia do TB atrapalha o desenvolvimento de tratamentos específicos (Geddes \& Miklowitz, 2013). 


\subsection{Fundamentação Teórica}

O medicamento estabilizador de humor ideal é aquele com eficácia antidepressiva e antimaníaca, sem induzir sintomas da polaridade oposta àquela em tratamento e que tenha eficácia na prevenção de novos episódios, tanto depressivos quanto maníacos. Quanto mais simples e com menos efeitos colaterais melhor é a aderência ao tratamento. Apesar disso, é comum a necessidade de se combinar o uso de dois ou mais estabilizadores de humor no tratamento de um episódio agudo ou mesmo no tratamento profilático. A seguir apresenta-se os principais tratamentos disponíveis de acordo com revisões (Geddes \& Miklowitz, 2013; Grunze et al., 2013; Yatham et al., 2013).

O lítio tem eficácia em torno de $70 \%$ no tratamento da mania, principalmente sem estado misto. A latência de resposta é de duas a três semanas. O lítio possui também moderado efeito antidepressivo, e é considerado o melhor estabilizador de humor no uso a longo prazo (Geddes \& Miklowitz, 2013) e na profilaxia do TB tipo I sem ciclagem rápida, com episódios bem delimitados e com boa recuperação interepisódica (tendo maior eficácia na profilaxia de episódios maníacos do que de episódios depressivos). É comercializado na forma de carbonato de lítio, e pode ser iniciado na dosagem de $600 \mathrm{mg} / \mathrm{dia}$, devendo-se titular a dosagem de acordo com a litemia (concentração sérica de lítio, que tem máxima eficácia em torno de 0,8 a 1,2 mEq/L). A coleta do sangue deve ser feita 4 a 7 dias após o início da terapia, e de 10-14h após a última tomada. A litemia deve ser repetida sempre que houver dúvidas sobre eficácia, toxicidade ou adesão. São contra-indicações absolutas à litioterapia: infarto agudo do miocárdio recente, arritmias cardíacas graves, psoríase e insuficiência renal aguda. Os efeitos colaterais mais comuns são tremor fino em mãos, náuseas (principalmente no início do tratamento), polidipsia, poliúria, ganho de peso e acne. Cerca de $20 \%$ dos 
pacientes desenvolvem hipotireoidismo subclínico a médio-longo prazo (aumento de TSH), o qual deve ser corrigido. Nefropatia induzida por lítio ocorre raramente, mas ocasionalmente pode ocasionar à suspensão do tratamento. Quando administrado em altas doses, o lítio pode prejudicar aprendizagem e memória (Geddes \& Miklowitz, 2013; Grunze et al., 2013; Yatham et al., 2013).

O valproato (valproato de sódio, divalproato de sódio, ácido valpróico) tem comprovada eficácia antimaníaca, principalmente nas manias e estados mistos, na ciclagem rápida, na comorbidade com transtornos ansiosos e abuso de álcool e substâncias. É a primeira opção, em alternativa ao lítio no tratamento da mania aguda. Pode haver rápida melhora do quadro clínico, em cerca de 1 semana, com a introdução e rápido aumento nas dosagens, (até de 20mg/kg). Níveis séricos entre 45 e $125 \mathrm{mcg} / \mathrm{mL}$ são necessários, de acordo com a tolerabilidade do paciente. Pode ser administrado em uma ou duas tomadas diárias, a depender da apresentação (liberação prolongada ou não). A eficácia antidepressiva é menor quando comparada ao lítio e na profilaxia é também mais eficaz na prevenção de episódios maníacos do que depressivos. Os efeitos colaterais mais comuns são náuseas, tremores dosedependentes, aumento de apetite e de peso, queda ou modificação da estrutura dos cabelos e discreta elevação de transaminases. Enzimas hepáticas e hemograma devem ser colhidos periodicamente, em função do risco (raro) de desenvolvimento de hepatotoxicidade aguda.

A carbamazepina tem eficácia antimaníaca, sobretudo em manias disfóricas (mistas) e ciclagem rápida, sendo considerada como agente de segunda opção para o tratamento. Pode ser administrada em duas tomadas diárias, com dosagens máximas em torno de $1200 \mathrm{mg} / \mathrm{dia}$ (níveis séricos adequados entre 4 e $12 \mathrm{mcg} / \mathrm{mL}$ ). Os efeitos colaterais mais comuns são 
redução benigna dos leucócitos, tontura, visão turva e sonolência (principalmente no início do tratamento). Enzimas hepáticas e hemograma devem ser colhidos periodicamente. A lamotrigina e o seu papel adjuvante no tratamento da depressão bipolar e na profilaxia dos episódios depressivos está em estudo. Não há evidência de eficácia no tratamento e prevenção de episódios maníacos. Pode ser administrada em uma tomada diária, com dosagens eficazes na faixa de 50 a $200 \mathrm{mg} / \mathrm{d}$ costuma ser bem tolerada, mas, devido ao risco de rash cutâneo (podendo nos casos graves evoluir para síndrome de Stevens-Johnson), a dosagem inicial deve ser no máximo de $25 \mathrm{mg} /$ dia, devendo ser aumentada lentamente. Ambas estas medicações podem ter como efeito colateral o prejuízo da atenção (Geddes \& Miklowitz, 2013).

Os antipsicóticos têm papel importante no manejo do TB, principalmente os antipsicóticos de segunda geração (ou "atípicos”), que podem apresentar efeitos mais rápidos do que litio e valproato na mania aguda. Os antipsicóticos de primeira geração têm eficácia comprovada na agitação e hiperatividade maníacas, mas devem ser utilizados em situações específicas devido ao risco de síndrome extrapiramidal e discinesia tardia e de depressão, no caso do haloperidol. Dentre os de segunda geração, quetiapina tem eficácia antimaníaca e antidepressiva bem estabelecidas. A risperidona, ziprazidona, aripiprazol, paliperidona e asenapina tiveram eficácia antimaníaca aguda comprovadas. A clozapina está reservada ao tratamento de casos refratários (tanto na mania aguda quanto na profilaxia), pelo risco de indução de agranulocitose. No uso de antipsicóticos de segunda geração, normalmente de custo mais alto, deve-se ter cuidado também com o risco de desenvolvimento de obesidade e síndrome metabólica (possíveis exceções são a ziprasidona e o aripiprazol). Antipsicóticos 
atípicos mais recentes são geralmente acompanhadas de pouco ou nenhum declínio cognitivo. (Geddes \& Miklowitz, 2013; Yatham et al., 2013).

O uso de antidepressivos deve ser evitado no tratamento do TB, principalmente tipo I, havendo evidências de que podem induzir episódios maníacos ou hipomaníacos e acelerar a ciclagem, piorando a evolução da doença. Seu uso deve ser reservado em associação com lítio, valproato e olanzapina ou para casos em que estratégias de primeira linha não tiveram eficácia no tratamento ou profilaxia de episódios depressivos, preferindo-se os inibidores seletivos de recaptura de serotonina (exceto paroxetina por evidências de baixa eficácia) ou a bupropiona pelo menor potencial de provocarem um episódio de mania. Devem ser descontinuados após 8 semanas da remissão da depressão e evitados no tratamento de estado misto ou pacientes com ciclagem rápida. Antidepressivos mais antigos, também chamados antidepressivos "tricíclicos" podem apresentar efeitos colaterais sobre a memória e habilidades executivas. (Geddes \& Miklowitz, 2013; Yatham et al., 2013).

Os benzodiazepínicos devem ser considerados quando se precisa de efeitos sedativos a curto prazo até que a medicação de escolha tenha tempo de fazer efeito. Esses medicamentos tendem a reduzir a atenção e capacidade de aprender novas informações, especialmente quando tomadas em altas doses, em pessoas idosas e em combinação com outras drogas (Yatham et al., 2013).

A eletroconvulsoterapia (ECT) é uma técnica que ainda não dispõe de estudos em número satisfatório e por causa disso está indicada na mania refratária como segunda linha, ou como terceira linha na depressão em TB I, sendo melhor indicada em depressões 
psicóticas, com estupor grave ou alto risco de suicídio. Esta intervenção pode apresentar como efeito colateral, prejuízos de memória e habilidades executivas (Yatham et al., 2013).

Diversos algoritmos e diretrizes para o tratamento do TB vêm sendo publicados por associações e grupos de especialistas. Em 2005, o Canadian Network for Mood and Anxiety Treatments (CANMAT) sintetizou as evidências disponíveis e definiu diretrizes de tratamento para o TB, posteriormente atualizadas em 2007, em 2009 (com a colaboração da ISBD) e em 2013 (Yatham et al., 2013), gerando uma das mais importantes diretrizes para o tratamento do TB. O CANMAT tem orientado as suas recomendações de acordo com os seguintes níveis de evidência científica: $1^{\text {a }}$ linha: meta-análise ou pelo menos dois estudos duplo-cegos, randomizados, controlados com placebo; ou pelo menos um estudo duplo-cego, randomizado, controlado com placebo e dados clínicos de eficácia e segurança. $2^{\mathrm{a}}$ linha: estudo aberto não-controlado com placebo ou comparador ativo com pelo menos 10 ou mais sujeitos e dados clínicos de eficácia e segurança. $3^{\text {a }}$ linha: relato de caso ou opinião de especialistas e dados clínicos de eficácia e segurança.

O tratamento farmacológico do TB é complexo e apresenta efeitos colaterais associados. Além disso, poucos medicamentos apresentam nível de evidência 1 de eficácia para o tratamento da depressão bipolar (Sienaert et al., 2012). O tratamento medicamentoso geralmente se concentra nos sintomas de depressão e mania e não em sintomas cognitivos. Outros estudos na literatura corroboram que alguns medicamentos usados para TB também prejudicam a cognição, como os anticonvulsivantes, lítio e antipsicóticos, que podem levar a retardo psicomotor, baixo desempenho de memória e prejuízos na memória verbal e na fluência verbal (Harvey et al., 2007; Malhi et al., 2016). 
O TB é uma doença que acomete o indivíduo por toda vida, com períodos de euforia e mais frequentemente depressão e é associada a importante risco familiar. Comorbidades clínicas e psiquiátricas são frequentes. O tratamento farmacológico é complexo e varia conforme a sintomatologia dos pacientes. Apesar dos inúmeros esforços feitos até o momento, ainda são necessárias novas perspectivas terapêuticas para melhorar a qualidade de vida desta população e de todos a eles ligados.

\subsection{Estimulação Transcraniana por Corrente Contínua}

Neste contexto, a Estimulação Transcraniana por Corrente Continua (ETCC) é uma nova intervenção terapêutica que vem apresentando um rápido desenvolvimento nos últimos anos, parecendo ser uma técnica promissora na terapêutica de vários distúrbios neuropsiquiátricos (Fregni \& Pascual-Leone, 2007; Brunoni et al., 2019). A técnica consiste em aplicar uma corrente elétrica contínua de baixa intensidade no cérebro via eletrodos colocados sob o escalpe. A corrente elétrica não gera potencial de ação nos próprios neurônios, mas facilita ou inibe a transmissão sináptica através do fluxo de elétrons que ocorre nos eletrodos na direção do cátodo ao ânodo (Nitsche et al., 2003; Brunoni et al., 2019).

Foi demonstrado que, utilizando doses, eletrodos e equipamentos adequados, uma quantia significativa de corrente elétrica atinge as redes neuronais (Miranda, Lomarev \& Hallett, 2006), levando à neuromodulação (Nitsche et al., 2003). O protocolo mais padronizado da técnica utiliza dois eletrodos de superfície - um servindo de cátodo e outro de ânodo. O ânodo é normalmente aplicado sobre a área do cérebro a qual se deseja estimular 
e o cátodo é aplicado ou no hemisfério oposto ou em uma solução salina. Aplica-se, então, uma corrente elétrica de 1-2mA com duração de tempo pré-determinado. Acredita-se que o ânodo exerça um efeito excitatório (potencial de repouso da membrana se altera, facilitando o processo de despolarização) sobre a região aplicada, enquanto que o cátodo exerce um efeito inibitório (potencial de repouso da membrana é hiperpolarizado, reduzindo disparo dos neurônios) nos neurônios desta região (Nitsche et al., 2008).

Alguns estudos translacionais já demonstraram a utilidade clínica deste método, por exemplo, em diminuir a fissura por cigarro (Rachid, 2016; Alghamdi et al., 2019), melhorar o desempenho cognitivo de idosos com doença de Alzheimer (Liu et al., 2017; Vacas et al., 2018) e diminuir a dor em pacientes com fibromialgia ( Roizenblatt et al., 2007; Duarte et al., 2018; Brighina et al., 2019). Os transtornos psiquiátricos também têm sido alvo desta ferramenta, porém a maioria dos estudos ainda contam com números amostrais pequenos (Tortella et al., 2015). Os mecanismos de ação por traz do efeito antidepressivo da ETCC ainda não são bem conhecidos. Mudanças em taxas de disparo de neurônios espontâneos e neuroplasticidade sináptica são as principais hipóteses para os efeitos que ocorrem no momento imediato e no momento após a estimulação. Portanto a técnica pode promover efeitos duradouros na atividade neuronal (Nitsche e Paulus, 2000), sem que seja induzido potenciais de ação durante a estimulação (Nitsche et al., 2008).

A estimulação por corrente contínua já havia sido utilizada em pacientes psiquiátricos na década de 1970 (Lolas, 1977), porém foi progressivamente abandonada com o surgimento de novos psicofármacos. Nos últimos anos a ETCC vem sendo bastante avaliada como uma técnica para a depressão unipolar. Estudos em depressão unipolar com amostras cada vez 
mais relevantes vem surgindo, como $\mathrm{n}=64$ (Loo et al., 2012), $\mathrm{n}=120$ (Brunoni et al., 2013) e $\mathrm{n}=289$ de uma meta-análise de dados de pacientes individuais (Brunoni et al., 2016; Martin et al., 2018), os quais revelaram resultados positivos para o tratamento. Brunoni et al. (2017), em um estudo com 245 pacientes adultos com depressão unipolar, demonstrou a não inferioridade da ETCC em relação ao tratamento com escitalopram. Loo et al., (2018) investigou a ETCC em 130 participantes com depressão uni e bipolar, não encontrando diferença antidepressiva entre estimulação ativa e simulada para ambos os casos. Por outro lado, uma meta-análise sugeriu que a ETCC também pode ser eficaz no tratamento da depressão em pacientes com TB (Dondé et al., 2018; Mutz et al., 2018). Com este campo sob investigação, uma ferramenta terapêutica eficaz e cognitivamente segura se torna importante para o cenário até o momento apresentado.

\subsection{Ensaio de Tratamento Elétrico de Depressão Bipolar [BETTER]}

O BETTER foi desenvolvido para ser um estudo de ensaio clínico aleatorizado, controlado por placebo (duplo cego), fase II/III para investigar os efeitos antidepressivos da ETCC na depressão bipolar. O projeto piloto foi iniciado em 2014 e sua apresentação premiada "tDCS in Bipolar Depression: A Piloty Study" no VI Simpósio Internacional em Neuromodulação, Universidade Presbiteriana Mackenzie, SP (Gabriel Tortella de Souza, Bernardo de Sampaio Pereira Junior, Roberta Casati e Andre Russowsky Brunoni) e posteriormente publicada por Pereira Junior et al., (2015). O mesmo estudo, então, caminhou em duas frentes: 1) a primeira, responsável por investigar a eficácia da ETCC como um 
tratamento para a depressão bipolar, foi tese de doutorado e publicada por Sampaio-Junior et al., (2018); 2) a segunda frente do estudo, a qual eu fui responsável por conduzir todas as etapas desde o início, foi responsável por investigar a segurança cognitiva da utilização da ETCC na depressão bipolar. Desta forma, avaliamos se a ETCC é uma alternativa terapeuticamente eficaz, com poucos efeitos colaterais e que não causa prejuízos cognitivos para esta população. Isto poderá trazer ganhos clínicos em curto prazo para pacientes que não toleram o uso de determinadas medicações, ou mostraram-se refratários aos mesmos. Em relação aos resultados da frente "1" (tratamento da depressão) do BETTER, observou-se que a ETCC apresentou melhora significativamente superior em relação ao grupo placebo, segurança e tolerabilidade da intervenção para sintomas de depressão (Sampaio-Junior et al., 2018). 


\section{MATERIAL E MÉTODOS}

\subsection{Objetivos}

O objetivo do estudo desta tese foi investigar se a ETCC é uma ferramenta terapêutica que apresenta segurança cognitiva no tratamento de pacientes com depressão bipolar. Para isso, foi usado como desfecho principal a mudança cognitiva nos testes neuropsicológicos entre o início e o final do estudo.

O objetivo exploratório foi avaliar se houve melhora cognitiva no grupo ETCC ativa. 


\subsection{Hipóteses}

A hipótese é que a ETCC Ativa quando comparada com a ETCC Simulada em pacientes com depressão bipolar, não apresentará diferença cognitiva entre os dois grupos, demonstrando assim que não houve declínio cognitivo com o uso da técnica. 


\subsection{Desenho do estudo}

Utilizou-se um ensaio clínico do tipo controlado, randomizado e duplo-cego. Pacientes elegíveis foram recrutados através de encaminhamentos de médicos de outros ambulatórios psiquiátricos da Universidade de São Paulo (USP), como por exemplo o GRUDA-IPqFMUSP (Grupo de doenças afetivas) e de divulgação externa do projeto de pesquisa, como e-mails, jornais internos e externos, websites e facebook. O estudo foi realizado no Hospital Universitário da USP, em São Paulo, Brasil, durante 1 ano e 8 meses, de $1^{\circ}$ de julho de 2014 a 30 de março de 2016. O estudo foi aprovado pelo Comitê de Ética Local e Nacional e registrado no clinicaltrials .gov (NCT02152878). Todos os participantes assinaram os formulários de consentimento informado.

Tabela 1. Fluxograma para cada paciente, ao longo do estudo.

\begin{tabular}{|c|c|c|c|c|c|}
\hline Atividade & Triagem & $\begin{array}{c}\text { Início } \\
\text { (Semana 0) }\end{array}$ & Semana 2 & Semana 4 & $\begin{array}{l}\text { Semana } 6 \\
\text { (EndPoint) }\end{array}$ \\
\hline $\begin{array}{c}\text { Critérios de Inclusão e } \\
\text { Exclusão }\end{array}$ & X & & & & \\
\hline MINI & $\mathrm{X}$ & & & & \\
\hline Termo de Consentimento & $X$ & & & & \\
\hline Sessão ETCC & & \multicolumn{2}{|c|}{10 sessões diárias } & $X$ & $X$ \\
\hline MADRS & & $\mathrm{X}$ & $X$ & $X$ & $\mathrm{X}$ \\
\hline HAMD17 & & $\mathrm{X}$ & X & $X$ & X \\
\hline BDI & & $\mathrm{X}$ & $\mathrm{X}$ & $X$ & $X$ \\
\hline
\end{tabular}




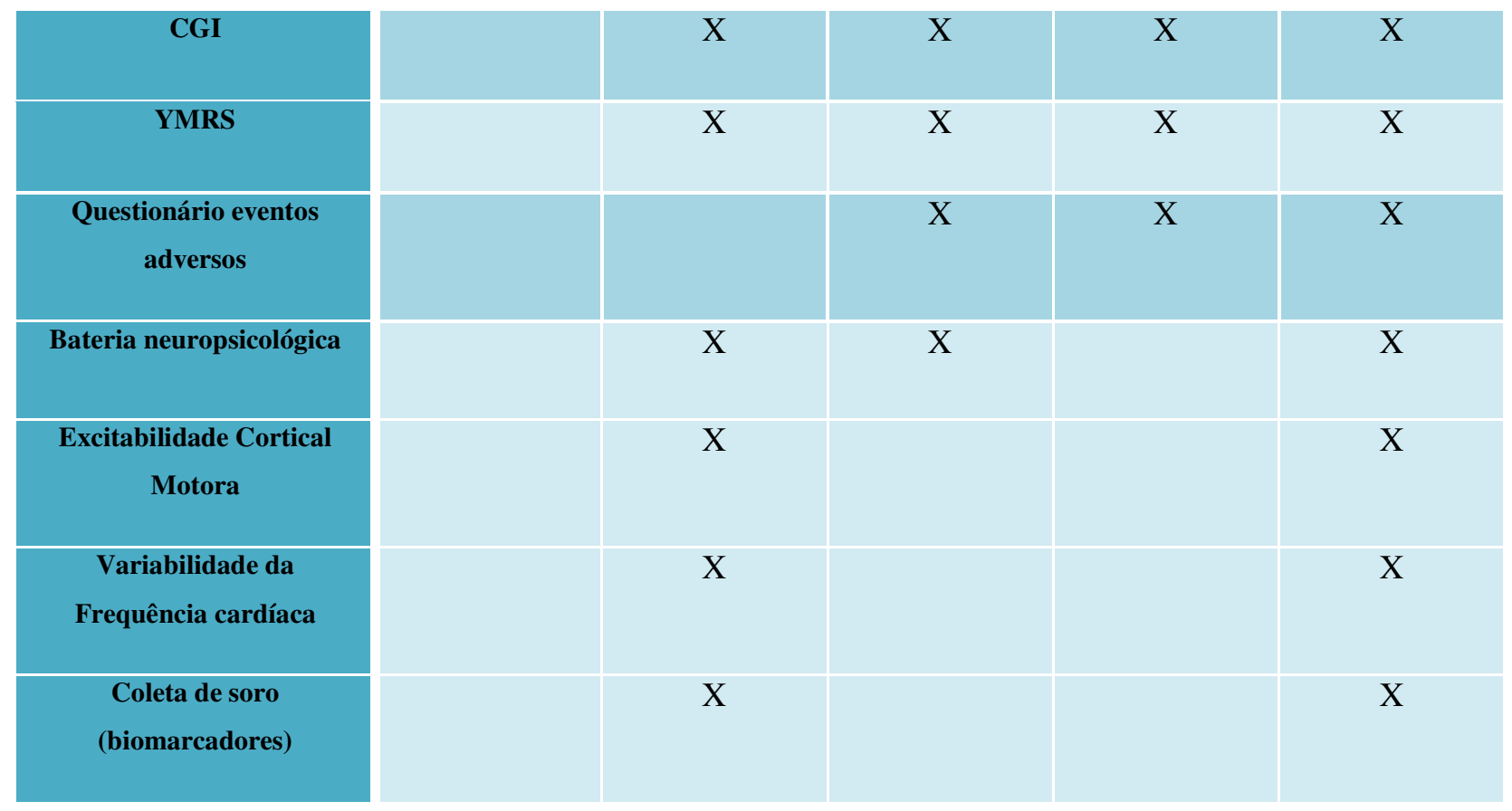

\section{Recrutamento}

Os voluntários interessados em participar do projeto de pesquisa respondiam inicialmente uma rápida triagem feita através de perguntas por e-mail (idade, diagnóstico prévio de TB e disponibilidade de participação). Os mesmos foram alocados para um dos grupos: grupo de estimulação placebo (ETCC Simulada) ou grupo ETCC ativa (ETCC Verdadeira). Os voluntários que não apresentaram melhora e tivessem sido alocados no grupo ETCC simulado puderam optar, voluntariamente, após o final do tratamento por receber 10 dias de estimulação ativa em ETCC. Por fim, os voluntários que não apresentarem melhora com ETCC ativa poderiam optar por participar de outras pesquisas com depressão bipolar (Estimulação magnética transcraniana profunda - bobina H1, em andamento naquele mesmo período pelo grupo de pesquisa) e futuramente, em outros protocolos de pesquisa que estiverem aprovados. 


\section{Randomização e Alocação}

Os participantes foram randomizados em dois grupos (ETCC placebo ou ETCC ativa) através de uma lista gerada em www.randomization.com usando randomização por blocos. A atribuição aos grupos realizou-se utilizando envelopes opacos selados contendo o código correspondente ao grupo designado para cada participante. Este código era imputado no dispositivo de ETCC que fornece automaticamente estimulação verdadeira ou placebo, sem o conhecimento da equipe.

\section{Critérios de Inclusão e de Exclusão}

Foram incluídos no estudo 59 adultos (18-65 anos), homens ou mulheres, bipolares diagnosticados, com sintomas deprimidos, apesar de um curso de tratamento adequado com estabilizadores de humor. Para inclusão, os mesmos deveriam apresentar um escore na Escala Hamilton para Depressão (HAMD-17) $\geq 17$, correspondente a um episódio depressivo de intensidade moderada a grave. Por fim, os pacientes deviam ser capazes de ler, compreender a língua portuguesa e ter pelo menos 6 anos de estudo.

Os critérios de exclusão foram: (1) outras condições neuropsiquiátricas, como a esquizofrenia, dependência de drogas, demências cerebrais, traumas cranianos, epilepsia e assim por diante (embora os participantes com distúrbios de ansiedade possam ser incluídos se o diagnóstico principal TB), (2) estados mistos com episódio (hipo)maníaco anterior(es) bem delimitados, definidos como apresentando simultaneamente sintomas maníacos, mensurados pela Escala de Avaliação de Mania de Young (YRMS > 8), ou episódios 
depressivos com sintomas mistos (pelo menos 3 características do polo oposto) mas sem episódios (hipo)maníacos prévios; (3) gravidez, (4) contra-indicações específicas para ETCC, (5) condições clínicas graves/fatais. Em relação ao uso de psicofármacos, estes deveriam estar em doses terapêuticas estáveis há no mínimo 4 semanas, para antidepressivos, e 2 semanas, para estabilizadores de humor. Drogas benzodiazepinicas foram permitidas apenas em doses baixas ( $\leq 10 \mathrm{mg} / \mathrm{dia}$ de diazepam ou equivalente). Os participantes poderiam, ainda, não estar fazendo uso de nenhuma medicação.

\section{Cegamento}

Este foi um estudo duplo-cego, ou seja, os pesquisadores, avaliadores e pacientes não tinham conhecimento quanto ao tratamento administrado até o termino do estudo. A técnica da ETCC é particularmente vantajosa para garantir o cegamento, quando comparada às demais técnicas de estimulação cerebral não invasiva. Isto porque a ETCC gera um leve formigamento na região da aplicação dos eletrodos, nos primeiros minutos da intervenção, não gerando efeitos adicionais posteriores. Utilizamos um aparelho de ETCC que permite programar um código em que o próprio aparelho realize o desligamento da corrente automaticamente, sem a necessidade de um operador. Para a ETCC placebo, o aparelho interrompe a aplicação da corrente 30 segundos após o seu início, portanto, imitando os efeitos colaterais iniciais na pele (Ex.: coceira, parestesia), porém sem induzir efeitos neuromoduladores. Com isto, garante-se, de fato, um duplo cegamento. 
Figura 1. Desligamento automático do equipamento simulado após trinta segundos.

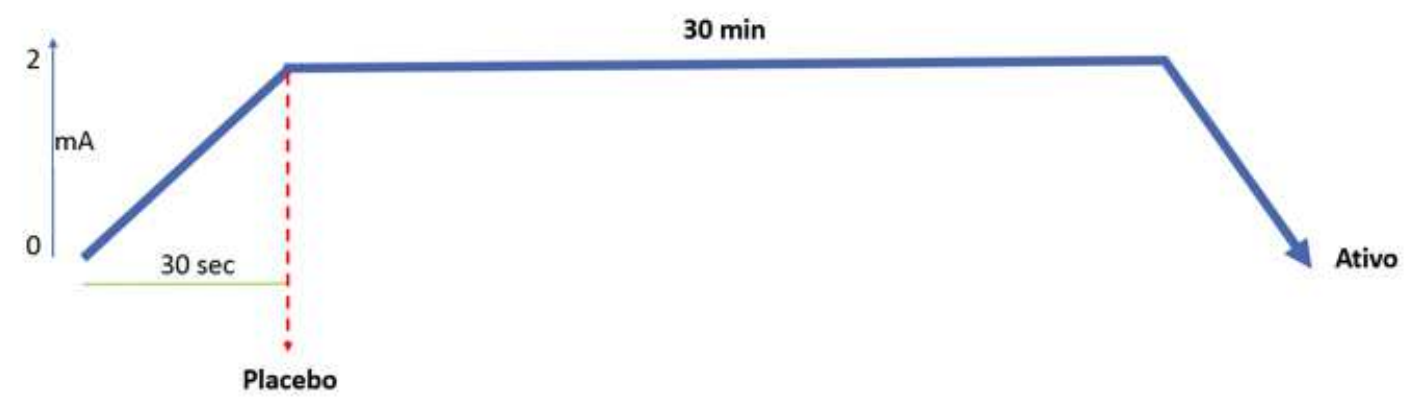

\section{Intervenções}

O modelo de equipamento utilizado foi um dispositivo ETCC (Soterix Medical, Nova York, NY, EUA, Modelo 1x1 tDCS-CT), que possui um teclado no qual um código de 6 dígitos é inserido para fornecer estimulação ativa ou simulada. Para a estimulação verdadeira, o ânodo e o catodo foram colocados respectivamente nas áreas cortical pré-frontal dorsolateral esquerda e direita (DLPFC). Este arranjo é conhecido por Bifrontal e já foi utilizado em estudos com depressão maior (Kalu et al., 2012). O método EASYstrap foi utilizado para posicionar os eletrodos (Seibt et al., 2015). Eletrodos de 5 x $5 \mathrm{~cm}$ e uma corrente de $2 \mathrm{~mA}$ foram utilizados por 30 minutos por dia. O ETCC foi aplicado por 10 dias consecutivos, com exceção dos finais de semana e, posteriormente, mais 2 vezes, um na semana 4 e, finalmente, na semana 6. 


\section{Variáveis clínicas}

O diagnóstico era confirmando utilizando-se a Mini International Neuropsychiatric Interview (MINI). A Montgomery- ̊̊sberg Depression Rating Scale (MADRS), YMRS, Escala de Impressão Clínica Global (CGI), HAMD-17 e Inventário de Depressão de Beck (BDI) foram aplicadas nas semanas 0, 2, 4 e 6. Concomitantemente, nas semanas 0, 2 e 6 foi realizada a avaliação cognitiva. Avaliou-se os efeitos colaterais utilizando um questionário padronizado (Brunoni et al., 2011). Todas as entrevistas clinicas, diagnosticas e de acompanhamento de humor foram realizadas por médicos psiquiatras com titulação e devidamente treinados para esse fim.

\section{Avaliação cognitiva}

Seguindo as diretrizes da Sociedade Internacional para Transtornos Bipolares - Bateria para Avaliação da Neurocognição (ISBD-BANC) (Yatham et al., 2010), uma bateria neuropsicológica foi construída. Os testes eram ministrados em uma única sessão e com duração aproximada de 80 minutos. A aplicação dos testes foi administrada a cada paciente no início do estudo (antes da primeira estimulação) e após 2 e 6 semanas (final do estudo). Os testes utilizados estão descritos abaixo e os domínios cognitivos avaliados estão organizados na Tabela 1.

- Teste de Fluência Verbal (Spreen \& Strauss, 1998): avalia função executiva, linguagem, fluência fonêmica e semântica associada às áreas frontais, atenção sustentada, planejamento, organização, julgamento/controle inibitório, estratégia e perseveração. 
Pede-se ao paciente para falar o maior número possível, que conseguir lembrar, de palavras que comecem primeiro com a letra $\mathrm{F}$, depois com A e $\mathrm{S}$, e para cada uma das letras, conta-se o tempo de um minuto. Não serão considerados nomes próprios (países, pessoas, marcas, etc.), derivados de uma mesma palavra (faca, faqueiro) e aumentativos e diminutivos (faquinha e facão).

- Fluência Verbal - Categoria Animal (fluência da linguagem por categoria semântica). Pede-se ao paciente para falar o maior número possível, que conseguir lembrar, de nomes de tipos de animais de qualquer espécie ou habitat e conta-se o tempo de um minuto.

- Teste de Memória Lógica das Duas Histórias (Kent, 2013): avalia memória auditivaverbal, memória lógica de armazenamento e recuperação. A parte 1 avalia a memória verbal imediata e a parte 2 (20’ minutos após a $1^{\circ}$ leitura) avalia memória tardia e capacidade de evocação. Baseia-se no exercício de retenção e evocação de duas histórias apresentadas oralmente (imediatamente após a leitura da história ao paciente o mesmo repete com o máximo de detalhes o que lembrar em relação à história contada).

- Cubos (subteste da Escala de Inteligência Wechsler para Adultos Terceira Edição: WAIS -III): avalia organização perceptual, conceitualização visuoespacial (componente espacial da percepção), nível conceitual e executivo (construção). O examinando deverá reproduzir modelos de duas cores com os cubos (cada cubo tem dois lados brancos, dois lados vermelhos, e dois lados metade vermelho e metade branco). O grau de dificuldade dos 
padrões é progressivo, com modelos compostos de dois cubos até os mais complexos com nove cubos.

- Teste de Aprendizagem Auditivo-Verbal de Rey - RAVLT (Magalhães et al., 2000): avalia aprendizagem, memória auditiva verbal, memória imediata de curto, e também de longo prazo, perseveração, intrusão, susceptibilidade à interferência, retenção após outra atividade e memória de reconhecimento. Fala-se uma lista de 15 palavras e pede-se para o examinando, logo em seguida, repetir todas que lembrar, não é necessário repetir na mesma ordem falada pelo avaliador. Esta lista é repetida 5 vezes, depois fala-se uma nova lista com 15 palavras diferentes (lista intrusiva) e pede-se para o examinando, logo em seguida, repetir todas que lembrar, não é necessário repetir na mesma ordem falada pelo avaliador. Em seguida, novamente, pede-se para o examinando tentar relembrar e dizer todas as palavras da $1^{\text {a }}$ lista que foi repetida 5 vezes anteriormente. Após 30 minutos pede-se para o examinando repetir novamente a $1^{\mathrm{a}}$ lista de palavras (memória a longo prazo). Após, o avaliador fala uma lista misturada de palavras para o paciente reconhecer quais palavras estavam na primeira lista (lista de reconhecimento).

- Teste de Stroop (Stroop Color Word Test - versão Vitória; Perret, 1974): avalia função executiva, atenção, flexibilidade do pensamento, atenção seletiva, resistência à interferência e impulsividade - controle inibitório. O teste consiste na apresentação de 3 cartelas em que o examinando deverá dizer o nome das cores o mais rápido que puder, sem segurar o cartão e nem apontar com o dedo (colocar o cartão sobre a mesa a frente do 
examinando). A primeira cartela tem cores (retângulos coloridos) e o examinando terá que dizer as cores na sequiência que os estímulos são apresentados (como se faz uma leitura, da esquerda para direita). A segunda cartela tem escritas palavras coloridas (nunca, cada, tudo e nada) e o examinando terá que dizer as cores das palavras na seqüência que os estímulos são apresentados (como se faz uma leitura, da esquerda para direita) e não o que está escrito. A terceira e última cartela (de interferência) tem escritos os nomes das cores pintadas (ex.: amarelo pintado de verde) e o examinando terá que dizer novamente as cores das palavras (nome das cores) na sequência que os estímulos são apresentados (como se faz uma leitura, da esquerda para direita) e não o que está escrito.

- Dígitos (subteste da escala verbal WAIS - III):

- Ordem Direta avalia: atenção, memória imediata (de curto prazo) e memória verbal.

- Ordem Inversa avalia: memória operacional (de trabalho), flexibilidade mental, concentração e vigilância. É um subteste composto por duas séries de dígitos que serão lidas pelo examinador e repetidas pelo paciente. A primeira série começa com 2 digitos, é lida pelo examinador na ordem direta e repetida pelo paciente na mesma ordem; a segunda série começa com 2 dígitos, é lida pelo examinador e repetida pelo paciente na ordem inversa.

- Teste das Trilhas - formas A e B (Trail Making - Reitan, 1958): avalia função executiva, atenção, acuidade visual, coordenação motora, velocidade de processamento; alternância, capacidade de alternar continuamente conceitos distintos, flexibilidade cognitiva, 
resistência à interferência, rapidez e tomada de decisão. A forma A avalia atenção, acuidade visual, coordenação motora e rapidez de processamento. Mostra-se a folha de exemplo da forma A e pede-se ao examinando que deverá ligar os números por linhas, as quais ele vai traçar. Depois do exemplo é apresentado uma outra folha com 25 círculos numerados e distribuídos ao acaso que devem ser unidos por uma linha contínua, como foi feito no exemplo. A forma B avalia alternância, capacidade de alternar continuamente conceitos distintos, flexibilidade cognitiva, resistência à interferência, rapidez e tomada de decisão. Mostra-se a folha de exemplo da forma B e explica-se ao examinando que deverá ligar, além dos números por linhas, as quais ele vai traçar, também terá que ligar as letras em ordem, alternando primeiro o número e depois a letra subseqüente. Ex.: ligar o número 1 à letra $\mathrm{A}, \mathrm{o} 2$ à $\mathrm{B}$ e assim sucessivamente. Depois de realizado o exemplo mostra-se a segunda folha com 13 números e 12 letras distribuídos ao acaso que devem ser unidos por uma linha contínua, como foi feito no exemplo.

- Códigos (subteste da escala de execução WAIS-III): avalia a performance psicomotora (persistência motora), atenção sustentada, velocidade de resposta e coordenação visuomotora, habilidade em decodificar símbolos, atenção seletiva, organização perceptiva e memória episódica. Apresentar folha impressa onde o examinando deverá copiar símbolos simples que estão associados com números. Usando uma chave (preenchimento: símbolos-números), o examinando desenha símbolos sob o número correspondente (exemplo) e depois começa o teste. Tempo: 2 minutos. 
Tabela 2. Bateria neuropsicológica utilizada.

\begin{tabular}{|c|c|c|c|}
\hline Domínio & Teste & Medida & Direção \\
\hline \multirow{4}{*}{$\begin{array}{l}\text { Memória de } \\
\text { trabalho e função } \\
\text { executiva }\end{array}$} & Trilhas-B (Reitan, 1955) & Tempo & Negativo \\
\hline & Cubos (Wechsler and Nascimento, 2004) & Número de respostas corretas & Positivo \\
\hline & Dígitos ordem indireta (Wechsler and Nascimento, 2004) & Amplitude máxima & Positivo \\
\hline & Stroop palavras (Perret, 1974) & Tempo & Negativo \\
\hline Controle initório & Stroop interferencia (Perret, 1974) & Tempo & Negativo \\
\hline \multirow{4}{*}{$\begin{array}{l}\text { Atenção e } \\
\text { velocidade de } \\
\text { processamento }\end{array}$} & Trilhas-A (Reitan, 1955) & Tempo & Negativo \\
\hline & Stroop cores (Perret, 1974) & Tempo & Negativo \\
\hline & Código (Wechsler and Nascimento, 2004) & Número de respostas corretas & Positivo \\
\hline & Dígitos ordem direta (Wechsler and Nascimento, 2004) & Amplitude máxima & Positivo \\
\hline \multirow[t]{2}{*}{ Memória } & RAVLT (Magalhães et al., 2010) & Número de palavras & Positivo \\
\hline & Memória lógica (Kent, 2013) & lembradas & Positivo \\
\hline Linguagem & Fluência verbal (Spreen e Strauss, 1998) & Número de palavras faladas & Positivo \\
\hline
\end{tabular}

\subsection{ANÁLISE ESTATÍSTICA}

Análises foram realizadas usando SAS® University Edition (2015). O primeiro passo foi converter os escores individuais dos pacientes em cada instrumento, assimetricamente distribuídos, em escores z normalizados (ie média $=0$, desvio padrão $=1$ ), obtidos através da formula: $z_{n}=\frac{x_{n}-\bar{X}}{\sigma_{x}}$ onde $z n$ é o valor padronizado da observação $n, x n$ é o valor original da observação $n$, e $\bar{X}$ e $\sigma_{n}$ são a média e o desvio padrão da variável $X$. A estratégia de usar variáveis compostas tem sido usada em estudos de neuromodulação (Schulze et al., 2016; Myczkowski et al., 2018 ) como uma tentativa de reduzir o número de resultados falsos positivos devido a comparações múltiplas e fornecer uma interpretação mais significativa dos aspectos cognitivos. Para construir os modelos mistos, foram agrupados os testes que avaliam as mesmas habilidades cognitivas, conforme apresentado na Tabela 2. Os escores foram obtidos para cinco grupos cognitivos avaliados (memória, memória operacional, atenção, 
linguagem e controle inibitório).

Uma análise de medidas repetidas usando o PROC MIXED foi conduzida considerando observações repetidas ao longo do tempo, considerando os fatores "Grupos", "Tempo" e "Grupos" vs. "Tempo", e significativo quando P <0,05. As correlações de Pearson foram realizadas entre a melhora da depressão e os grupos cognitivos para explorar as correlações entre essas variáveis. As variáveis dependentes consideradas foram os domínios cognitivos, enquanto as variáveis independentes consideradas foram grupos (dois níveis: ETCC e placebo) e tempo (três níveis: baseline, semana 4 e semana 6). O tamanho da amostra foi estimado para uma potência de $80 \%$ e um nível $\alpha$ bi caudal de $5 \%$ para os desfechos clínicos apresentados em Sampaio-Junior et al (2018). Calculamos com base nos estudos de Brunoni et al. (2013) e Kalu et al. (2012) disponível quando este estudo foi concebido obtendo-se um total de amostras de 55 e 52 participantes, respectivamente. Considerou-se uma taxa de atrito de $10 \%$ a $15 \%$, aumentando o tamanho da amostra para 58 a 60 participantes. 


\section{RESULTADOS}

De um total inicial de 912 voluntários, 221 foram triados e destes 162 foram excluídos.

Dos 59 pacientes incluídos no estudo, houveram 3 dropouts no grupo placebo e 4 dropouts no grupo ativo, concluindo o estudo 52 participantes. O fluxograma detalhado se encontra abaixo:

Figura 2. Fluxograma dos pacientes encaminhados e incluídos no estudo.

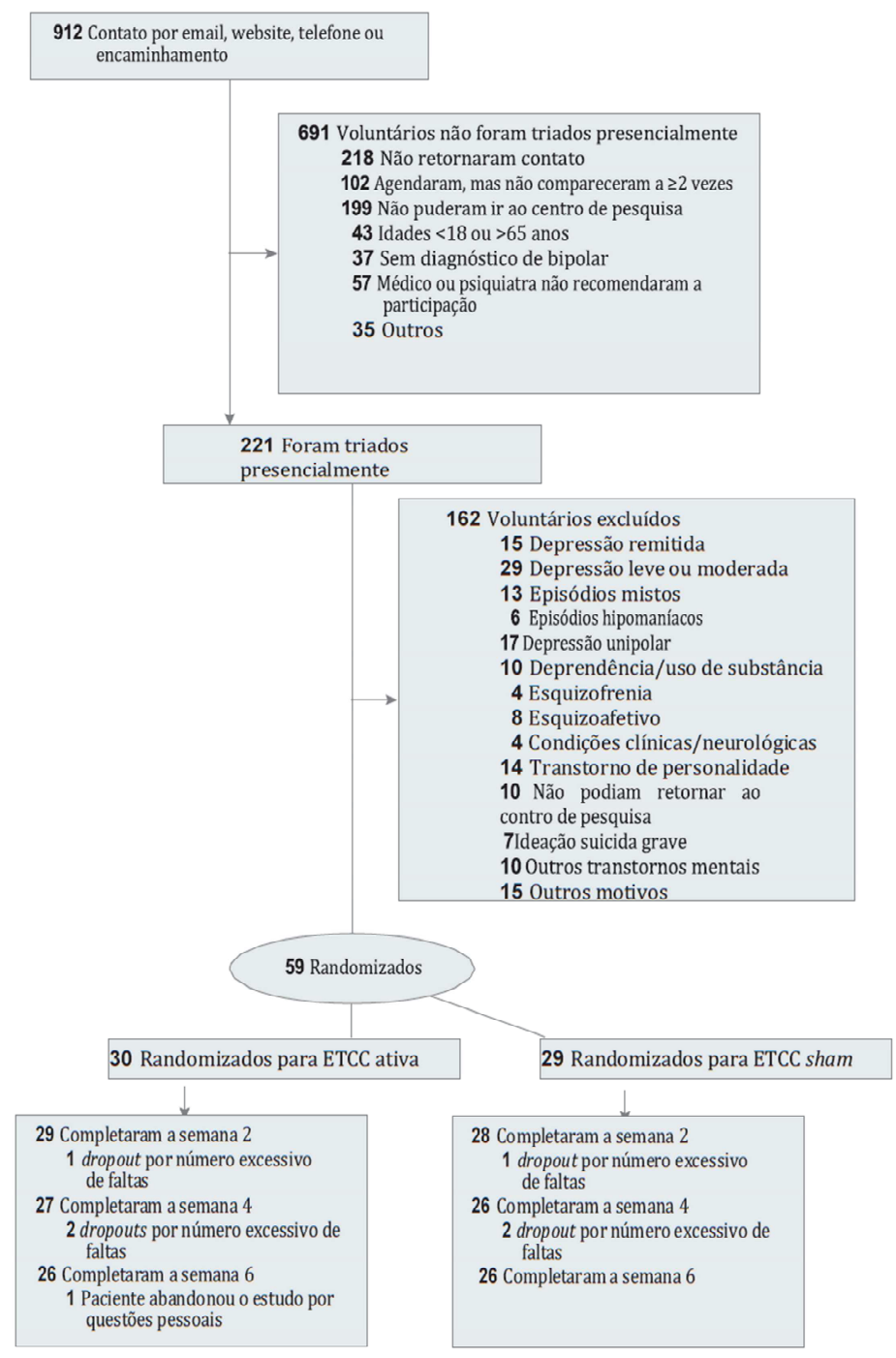


As variáveis clínicas e demográficas no início do estudo estão descritas na Tabela 3. Apenas as variáveis sexo (mais mulheres no grupo placebo) e etnia (mais não brancos no grupo placebo) apresentaram diferença estatisticamente significante entre os grupos no início do estudo. Todas as outras variáveis foram distribuídas de forma homogênea.

Tabela 3. Características clínicas e demográficos da amostra no início do estudo.

\begin{tabular}{|c|c|c|c|c|}
\hline & $\begin{array}{l}\text { Placebo } \\
(n=29)\end{array}$ & $\begin{array}{l}\text { ETCC } \\
(n=30)\end{array}$ & $p$ & $\begin{array}{c}\text { Total } \\
(n=59)\end{array}$ \\
\hline \multicolumn{5}{|c|}{ Características clínicas e demográficos } \\
\hline Gênero (\% Fem) & $24(82.76)$ & $16(53.33)$ & 0.01 & $\begin{array}{c}40 \\
(67.80)\end{array}$ \\
\hline Idade, média (SD) & $\begin{array}{c}45.72 \\
(10.26)\end{array}$ & $\begin{array}{c}46.16 \\
(11.75)\end{array}$ & 0.87 & $\begin{array}{c}45.94 \\
(11.95)\end{array}$ \\
\hline Anos de escolaridade, média (SD) & $\begin{array}{l}17.43 \\
(6.57)\end{array}$ & $15.71(4.01)$ & 0.3 & $\begin{array}{l}16.61 \\
(5.50)\end{array}$ \\
\hline Renda ( $\%<5$ S.M.) & $11(50)$ & $7(33.33)$ & 0.26 & $1841(86)$ \\
\hline Etnia (\% Brancos) & $15(51.72)$ & $23(76.67)$ & 0.04 & $\begin{array}{c}38 \\
(64.41)\end{array}$ \\
\hline Estado Civil (\% solteiros) & $17(58.52)$ & $11(36.67)$ & 0.09 & $\begin{array}{c}28 \\
(47.46) \\
\end{array}$ \\
\hline Índice de massa corporal, média (SD) & $27.7(7.9)$ & $27.8(5.0)$ & 0.9 & $27.7(6.5)$ \\
\hline \multicolumn{5}{|c|}{ Características da Depressão } \\
\hline Idade de início, média (SD) & $\begin{array}{c}20.2 \\
(8.09) \\
\end{array}$ & $23.5(11.18)$ & 0.20 & $\begin{array}{c}21.8 \\
(9.84) \\
\end{array}$ \\
\hline Bipolar tipo I, n (\%) & $16(55.17)$ & $20(66.67)$ & 0.36 & $\begin{array}{c}36 \\
(61.02)\end{array}$ \\
\hline Bipolar tipo II, n (\%) & $13(44.83)$ & $10(33.33)$ & 0.36 & $23(38.98)$ \\
\hline Número de episódios, média (SD) & $\begin{array}{c}17.6 \\
(10.8)\end{array}$ & $15.2(12.1)$ & 0.41 & $\begin{array}{c}16.4 \\
(11.4)\end{array}$ \\
\hline$>12$ duração meses, $\mathrm{n}(\%)$ & $8(27.59)$ & $10(33.33)$ & 0.63 & $\begin{array}{c}18 \\
(30.51)\end{array}$ \\
\hline Severidade da depressão, n (\%) & $14(40.28)$ & $14(40.67)$ & 0.90 & $\begin{array}{c}28 \\
(47.46)\end{array}$ \\
\hline \multicolumn{5}{|c|}{ Escores semana 0 , média (SD) } \\
\hline HDRS17 & $\begin{array}{l}23.48 \\
(4.74) \\
\end{array}$ & $23.06(3.93)$ & 0.71 & $\begin{array}{l}23.27 \\
(4.31) \\
\end{array}$ \\
\hline MADRS & $\begin{array}{l}27.83 \\
(4.95)\end{array}$ & $28.66(5.33)$ & 0.58 & $\begin{array}{l}28.30 \\
(5.12)\end{array}$ \\
\hline CGI & $\begin{array}{c}3.41 \\
(0.68)\end{array}$ & $3.46(0.77)$ & 0.78 & $\begin{array}{c}3.44 \\
(0.72)\end{array}$ \\
\hline BDI & $\begin{array}{l}33.85 \\
(8.37)\end{array}$ & $31.83(9.30)$ & 0.38 & $\begin{array}{l}32.81 \\
(8.84)\end{array}$ \\
\hline YMRS & $2(1.87)$ & $2.4(2.06)$ & 0.43 & $\begin{array}{c}2.20 \\
(1.96)\end{array}$ \\
\hline
\end{tabular}




\begin{tabular}{lcccc}
\hline Falha de tratamento (atual), média (SD) & $\begin{array}{c}2.10 \\
(1.28)\end{array}$ & $2.28(1.16)$ & 0.57 & $\begin{array}{c}2.19 \\
(1.21)\end{array}$ \\
\hline Falha de tratamento (vida), média (SD) & $\begin{array}{c}9.34 \\
(4.04)\end{array}$ & $8.65(4.40)$ & 0.53 & $\begin{array}{c}8.99 \\
(4.21)\end{array}$ \\
\hline Resistente ao tratamento, n (\%) & $20(68.97)$ & $17(56.67)$ & 0.32 & $\begin{array}{c}37 \\
(62.71)\end{array}$ \\
\hline Benzodiazepínicos, n (\%) & $16(55.1)$ & $11(36.6)$ & 0.15 & $\begin{array}{c}27 \\
(45.76)\end{array}$ \\
\hline SSRIs, n (\%) & $14(48.28)$ & $9(30)$ & 0.11 & $\begin{array}{c}23 \\
(38.98)\end{array}$ \\
\hline Qualquer medicamento antidepressivo & $23(79.31)$ & $21(70)$ & 0.41 & $\begin{array}{c}44 \\
(74.58)\end{array}$ \\
\hline Velanfaxina & $2(6.90)$ & $4(13.33)$ & 0.67 & $6(10.17)$ \\
\hline Bupropriona & $4(13.79)$ & $4(13.33)$ & 1 & $8(13.56)$ \\
\hline Lítio & $7(24.14)$ & $13(43.33)$ & 0.12 & $\begin{array}{c}20 \\
\text { Qulproato }\end{array}$ \\
\hline Lamotrigina & $9(31.03)$ & $9(30)$ & 0.93 & $\begin{array}{c}18 \\
\text { Quetiapina }\end{array}$ \\
\hline
\end{tabular}

Tabela 4. Escores dos testes neuropsicológicos ao longo do tempo em grupos ativos e simulados.

\begin{tabular}{|c|c|c|c|c|c|c|}
\hline & \multicolumn{2}{|l|}{ Início } & \multicolumn{2}{|c|}{ Semana 2} & \multicolumn{2}{|c|}{ Semana 6} \\
\hline & Ativo & Placebo & Ativo & Placebo & Ativo & Placebo \\
\hline $\begin{array}{l}\text { RAVLT, média } \\
\text { (SD) }\end{array}$ & $\begin{array}{l}41.60 \\
(12.01)\end{array}$ & $\begin{array}{l}42.86 \\
(8.56)\end{array}$ & $\begin{array}{l}50.64 \\
(11.67)\end{array}$ & $\begin{array}{l}47.93 \\
(9.92)\end{array}$ & $\begin{array}{l}49.33 \\
(15.70)\end{array}$ & $\begin{array}{l}47.12 \\
(15.75)\end{array}$ \\
\hline $\begin{array}{l}\text { Memória } \\
\text { lógica, média } \\
\text { (SD) }\end{array}$ & $\begin{array}{l}36.79 \\
(12.94)\end{array}$ & $\begin{array}{l}35.57 \\
(11.83)\end{array}$ & $\begin{array}{l}39.57 \\
(13.18)\end{array}$ & $\begin{array}{l}41.22 \\
(13.64)\end{array}$ & $\begin{array}{l}46.50 \\
(14.03)\end{array}$ & $\begin{array}{l}41.76 \\
(14.01)\end{array}$ \\
\hline $\begin{array}{l}\text { Teste de } \\
\text { fluência verbal, } \\
\text { média (SD) }\end{array}$ & $\begin{array}{l}31.73 \\
(9.12)\end{array}$ & $\begin{array}{l}33.21 \\
(10.51)\end{array}$ & $\begin{array}{l}33.03 \\
(11.35)\end{array}$ & $\begin{array}{l}31.78 \\
(11.38)\end{array}$ & $\begin{array}{l}35.08 \\
(10.86)\end{array}$ & $\begin{array}{l}36.12 \\
(13.17)\end{array}$ \\
\hline $\begin{array}{l}\text { Cubos, média } \\
\text { (SD) }\end{array}$ & $\begin{array}{l}30.63 \\
(14.82)\end{array}$ & $\begin{array}{l}29.76 \\
(12.17)\end{array}$ & $\begin{array}{l}32.14 \\
(14.26)\end{array}$ & $\begin{array}{l}32.63 \\
(9.54)\end{array}$ & $\begin{array}{l}31.27 \\
(14.47)\end{array}$ & $\begin{array}{l}35.44 \\
(12.54)\end{array}$ \\
\hline $\begin{array}{l}\text { Stroop cores, } \\
\text { média (SD) }\end{array}$ & $\begin{array}{l}14.45 \\
(2.82)\end{array}$ & $\begin{array}{l}15.04 \\
(3.90)\end{array}$ & $\begin{array}{l}14.36 \\
(2.84)\end{array}$ & $\begin{array}{l}14.35 \\
(3.73)\end{array}$ & $\begin{array}{l}14.56 \\
(3.44)\end{array}$ & $\begin{array}{l}14.33 \\
(3.57)\end{array}$ \\
\hline
\end{tabular}




\begin{tabular}{|c|c|c|c|c|c|c|}
\hline $\begin{array}{l}\text { Stroop } \\
\text { palavras, média } \\
\text { (SD) }\end{array}$ & $\begin{array}{l}19.66 \\
(4.62)\end{array}$ & $\begin{array}{l}19.25 \\
(5.67)\end{array}$ & $\begin{array}{l}18.43 \\
(4.17)\end{array}$ & $\begin{array}{l}18.62 \\
(5.48)\end{array}$ & $\begin{array}{l}19.56 \\
(5.96)\end{array}$ & $\begin{array}{l}17.33 \\
(4.06)\end{array}$ \\
\hline $\begin{array}{l}\text { Stroop } \\
\text { interferência, } \\
\text { média (SD) }\end{array}$ & $\begin{array}{l}28.55 \\
(7.40)\end{array}$ & $\begin{array}{l}31.43 \\
(16.57)\end{array}$ & $\begin{array}{l}27.43 \\
(9.68)\end{array}$ & $\begin{array}{l}27.23 \\
(9.92)\end{array}$ & $\begin{array}{l}28.08 \\
(11.92)\end{array}$ & $\begin{array}{l}26.04 \\
(8.77)\end{array}$ \\
\hline $\begin{array}{l}\text { Dígitos, ordem } \\
\text { direta, média } \\
\text { (SD) }\end{array}$ & $8.13(2.42)$ & $7.48(2.10)$ & $\begin{array}{l}8.11 \\
(2.23)\end{array}$ & $\begin{array}{l}7.52 \\
(1.58)\end{array}$ & $\begin{array}{l}7.92 \\
(2.36)\end{array}$ & $\begin{array}{l}7.92 \\
(2.17)\end{array}$ \\
\hline $\begin{array}{l}\text { Dígitos, ordem } \\
\text { indireta, média } \\
\text { (SD) }\end{array}$ & $5.60(1.92)$ & $5.59(2.04)$ & $\begin{array}{l}6.00 \\
(1.87)\end{array}$ & $\begin{array}{l}5.67 \\
(2.09)\end{array}$ & $\begin{array}{l}5.84 \\
(1.97)\end{array}$ & $\begin{array}{l}5.21 \\
(2.25)\end{array}$ \\
\hline $\begin{array}{l}\text { Trilhas-A, } \\
\text { média (SD) }\end{array}$ & $\begin{array}{l}40.59 \\
(14.95)\end{array}$ & $\begin{array}{l}41.38 \\
(12.20)\end{array}$ & $\begin{array}{l}45.36 \\
(21.18)\end{array}$ & $\begin{array}{l}47.67 \\
(23.10)\end{array}$ & $\begin{array}{l}42.54 \\
(28.62)\end{array}$ & $\begin{array}{l}37.04 \\
(12.80)\end{array}$ \\
\hline $\begin{array}{l}\text { Trilhas-B, } \\
\text { média (SD) }\end{array}$ & $\begin{array}{l}112.28 \\
(68.48)\end{array}$ & $\begin{array}{l}112.38 \\
(49.55)\end{array}$ & $\begin{array}{l}96.46 \\
(49.44)\end{array}$ & $\begin{array}{l}96.70 \\
(46.06)\end{array}$ & $\begin{array}{l}93.73 \\
(44.18)\end{array}$ & $\begin{array}{l}88.00 \\
(35.83)\end{array}$ \\
\hline $\begin{array}{l}\text { Códigos, } \\
\text { média (SD) }\end{array}$ & $\begin{array}{l}51.93 \\
(14.29)\end{array}$ & $\begin{array}{l}54.83 \\
(15.94)\end{array}$ & $\begin{array}{l}56.93 \\
(16.36)\end{array}$ & $\begin{array}{l}56.85 \\
(14.22)\end{array}$ & $\begin{array}{l}54.58 \\
(18.41)\end{array}$ & $\begin{array}{l}60.92 \\
(15.96)\end{array}$ \\
\hline
\end{tabular}

A Tabela 4. apresentada anteriormente apresenta os escores obtidos nos testes cognitivos ao longo do estudo em ambos os grupos. A Tabela 5 mostra o desempenho cognitivo de cada teste, por grupo, na semana 0 e na semana 6.

Tabela 5. Características cognitivas homogênea da amostra no início e final do estudo.

\begin{tabular}{|c|c|c|c|c|c|}
\hline & & $\begin{array}{c}\text { Placebo } \\
(\mathrm{n}=29)\end{array}$ & $\begin{array}{l}\text { ETCC } \\
(\mathrm{n}=30)\end{array}$ & $p$ & Total $(n=59)$ \\
\hline \multicolumn{6}{|l|}{ Características Cognitivas } \\
\hline \multirow[t]{2}{*}{ RAVLT, média (SD) } & Semana 0 & $42(8.56)$ & $41(12.01)$ & 0.64 & $42(10.38)$ \\
\hline & Semana 6 & $\begin{array}{c}47 \\
(15.74)\end{array}$ & $49(15.69)$ & 0.60 & $48(15.60)$ \\
\hline \multirow[t]{2}{*}{ Memória Lógica, média (SD) } & Semana 0 & $\begin{array}{c}35 \\
(11.83)\end{array}$ & $36(12.93)$ & 0.71 & $36(12.29)$ \\
\hline & Semana 6 & $\begin{array}{c}41 \\
(14.01) \\
\end{array}$ & $46(14.03)$ & 0.23 & $44(14.08)$ \\
\hline Fluência Verbal, média (SD) & Semana 0 & $\begin{array}{c}33 \\
(10.50)\end{array}$ & $31(9.11)$ & 0.56 & $32(9.76)$ \\
\hline
\end{tabular}




\begin{tabular}{|c|c|c|c|c|c|}
\hline & Semana 6 & $\begin{array}{c}36 \\
(13.16)\end{array}$ & $35(10.86)$ & 0.75 & $35(11.93)$ \\
\hline \multirow[t]{2}{*}{ Cubos, média (SD) } & Semana 0 & $\begin{array}{c}29 \\
(12.16) \\
\end{array}$ & $30(14.81)$ & 0.8 & $30(13.46)$ \\
\hline & Semana 6 & $\begin{array}{c}35 \\
(12.53)\end{array}$ & $31(14.46)$ & 0.27 & $33(13.58)$ \\
\hline \multirow[t]{2}{*}{ Stroop1, média (SD) } & Semana 0 & $15(3.90)$ & $14(2.82)$ & 0.51 & $14(3.37)$ \\
\hline & Semana 6 & $14(3.57)$ & $14(3.44)$ & 0.82 & $14(3.47)$ \\
\hline \multirow[t]{2}{*}{ Stroop2, média (SD) } & Semana 0 & $19(5.67)$ & $19(4.61)$ & 0.76 & $19(5.12)$ \\
\hline & Semana 6 & $17(4.06)$ & $19(5.95)$ & 0.13 & $18(5.18)$ \\
\hline \multirow[t]{2}{*}{ Stroop interferência, média (SD) } & Semana 0 & $\begin{array}{c}31 \\
(16.57)\end{array}$ & $28(7.40)$ & 0.39 & $29(12.72)$ \\
\hline & Semana 6 & $26(8.77)$ & $28(11.91)$ & 0.5 & $27(10.43)$ \\
\hline \multirow[t]{2}{*}{ Dígitos, média (SD) } & Semana 0 & $13(3.77)$ & $13(3.73)$ & 0.49 & $13(3.73)$ \\
\hline & Semana 6 & $12(4.77)$ & $13(4.04)$ & 0.35 & $13(4.42)$ \\
\hline \multirow[t]{2}{*}{ TMT-A, média (SD) } & Semana 0 & $\begin{array}{c}41 \\
(12.20)\end{array}$ & $40(14.94)$ & 0.82 & $40(13.53)$ \\
\hline & Semana 6 & $\begin{array}{c}37 \\
(12.80)\end{array}$ & $42(28.61)$ & 0.38 & $39(22.26)$ \\
\hline \multirow[t]{2}{*}{ TMT-B, média (SD) } & Semana 0 & $\begin{array}{c}112 \\
(49.54) \\
\end{array}$ & $\begin{array}{c}112 \\
(68.47) \\
\end{array}$ & 0.99 & $112(59.24)$ \\
\hline & Semana 6 & $\begin{array}{c}37 \\
(12.80)\end{array}$ & $42(28.61)$ & 0.38 & $39(22.26)$ \\
\hline \multirow[t]{2}{*}{ Códigos, média (SD) } & Semana 0 & $\begin{array}{c}54 \\
(15.93) \\
\end{array}$ & $51(14.28)$ & 0.47 & $53(15.08)$ \\
\hline & Semana 6 & $\begin{array}{c}60 \\
(15.95)\end{array}$ & $54(18.40)$ & 0.19 & $57(17.37)$ \\
\hline
\end{tabular}

Não houve diferença significativa entre o grupo placebo e o ETCC no início e no final do estudo, para nenhuma das características cognitivas. Além disso, não houve diferenças significativas entre a linha de base e 6 semanas após a submissão aos tratamentos Placebo e ETCC para Fluência Verbal, Stroop cores, Stroop palavras, Stroop interferência, Dígitos, TMT-A, TMT-B e Códigos. Por sua vez, 6 semanas após o início do estudo, os pacientes tiveram pontuações RAVLT maiores do que as observadas na linha de base $(\mathrm{P} \leq 0,05)$, nos grupos Placebo e ETCC. O teste de Memória Lógica apresentou alteração significativa apenas para o grupo ETCC, mas não para o grupo Placebo, enquanto o teste Cubos apresentou 
alteração significativa apenas para o grupo Placebo e não para ETCC, entre o início do estudo e a $6^{\mathrm{a}}$ semanas (Tabela 6 ).

Tabela 6. Diferença entre o grupo placebo e o ativo no início e no final do estudo.

\begin{tabular}{|c|c|c|c|c|}
\hline & & $\begin{array}{l}\text { Placebo } \\
(n=29)\end{array}$ & Ativo $(n=30)$ & Total $(n=59)$ \\
\hline \multirow{2}{*}{ RAVLT, média (SD) } & 0 & $42(8.56)$ & $41(12.01)$ & $42(10.38)$ \\
\hline & 6 & $47(15.74)$ & 49 (15.69) & $48(15.60)$ \\
\hline Diferença $(p)$ & & $5(\mathbf{0 . 0 5})$ & $8(<0.01)$ & \\
\hline \multirow{2}{*}{ Memória lógica, média (SD) } & 0 & $35(11.83)$ & $36(12.93)$ & $36(12.29)$ \\
\hline & 6 & $41(14.01)$ & $46(14.03)$ & $44(14.08)$ \\
\hline Diferença $(p)$ & & $6(0.12)$ & $10(\mathbf{0 . 0 2})$ & \\
\hline \multirow{2}{*}{ Fluência verbal, média (SD) } & 0 & $33(10.50)$ & $31(9.11)$ & $32(9.76)$ \\
\hline & 6 & $36(13.16)$ & $35(10.86)$ & $35(11.93)$ \\
\hline Diferença $(p)$ & & $3(0.09)$ & $4(0.07)$ & \\
\hline \multirow[b]{2}{*}{ Cubos, média (SD) } & 0 & $29(12.16)$ & $30(14.81)$ & $30(13.46)$ \\
\hline & 6 & $35(12.53)$ & $31(14.46)$ & $33(13.58)$ \\
\hline Diferença $(p)$ & & $6(\mathbf{0 . 0 3})$ & $1(2.35)$ & \\
\hline \multirow{2}{*}{ Stroop cores, média (SD) } & 0 & $15(3.90)$ & $14(2.82)$ & $14(3.37)$ \\
\hline & 6 & $14(3.57)$ & $14(3.44)$ & $14(3.47)$ \\
\hline Diferença $(p)$ & & $1(0.47)$ & $0(0.91)$ & \\
\hline \multirow{2}{*}{ Stroop palavras, média (SD) } & 0 & $19(5.67)$ & $19(4.61)$ & $19(5.12)$ \\
\hline & 6 & $17(4.06)$ & $19(5.95)$ & $18(5.18)$ \\
\hline Diferença $(p)$ & & $2(0.18)$ & $0(0.95)$ & \\
\hline \multirow{2}{*}{ Stroop interferência, média (SD) } & 0 & $31(16.57)$ & $28(7.40)$ & $29(12.72)$ \\
\hline & 6 & $26(8.77)$ & $28(11.91)$ & $27(10.43)$ \\
\hline Diferença $(p)$ & & $5(0.10)$ & $0(0.88)$ & \\
\hline \multirow{2}{*}{ Dígitos, média (SD) } & 0 & $13(3.77)$ & $13(3.73)$ & $13(3.73)$ \\
\hline & 6 & $12(4.77)$ & $13(4.04)$ & $13(4.42)$ \\
\hline
\end{tabular}




\begin{tabular}{|c|c|c|c|c|}
\hline Diferença $(p)$ & & $1(0.96)$ & $0(0.98)$ & \\
\hline \multirow{2}{*}{ TMT-A, média (SD) } & 0 & $41(12.20)$ & $40(14.94)$ & $40(13.53)$ \\
\hline & 6 & $37(12.80)$ & $42(28.61)$ & $39(22.26)$ \\
\hline Diferença $(p)$ & & $4(0.38)$ & $2(0.69)$ & \\
\hline \multirow{2}{*}{ TMT-B, média (SD) } & 0 & $112(49.54)$ & $112(68.47)$ & $112(59.24)$ \\
\hline & 6 & $37(12.80)$ & $42(28.61)$ & $39(22.26)$ \\
\hline Diferença $(p)$ & & $75(0.09)$ & $70(0.19)$ & \\
\hline \multirow{2}{*}{ Códigos, média (SD) } & 0 & $54(15.93)$ & $51(14.28)$ & $53(15.08)$ \\
\hline & 6 & $60(15.95)$ & $54(18.40)$ & $57(17.37)$ \\
\hline Diferença $(p)$ & & $6(0.17)$ & $3(0.55)$ & \\
\hline
\end{tabular}

Foi encontrado um resultado significativo para o efeito do tempo de memória e controle inibitório (Tabela 7), mas não para o efeito de grupo. Não foram observados resultados significativos para a interação tempo vs grupo. Observamos que as médias dos escores tinham um alto desvio padrão nessa população, e isso pode ter interferido em achados significativos (Figura 2).

Tabela 7. Resultados do modelo misto para os efeitos principais e de interação dos fatores tempo, grupo, tempo x grupo e depressão.

\begin{tabular}{|c|c|c|c|c|c|c|}
\hline & \multicolumn{2}{|l|}{ Grupo } & \multicolumn{2}{|l|}{ Tempo } & \multicolumn{2}{|c|}{ Tempo vs Grupo } \\
\hline & F Value & $p$ & F Value & $p$ & F Value & $p$ \\
\hline Memória & 0.16 & 0.6865 & 10.27 & $<.0001$ & 0.46 & 0.6321 \\
\hline Memória de trabalho & 0.28 & 0.6003 & 0.94 & 0.3928 & 0.44 & 0.6457 \\
\hline Atenção & 0.51 & 0.4781 & 1.71 & 0.1856 & 0.36 & 0.7010 \\
\hline Linguagem & 0.07 & 0.7926 & 2.05 & 0.1333 & 0.59 & 0.5578 \\
\hline Controle inibitório & 0.00 & 0.9856 & 3.21 & 0.0443 & 2.78 & 0.0665 \\
\hline
\end{tabular}


Figura 3. Alterações cognitivas ao longo do tempo em grupos ativo vs. simulado.
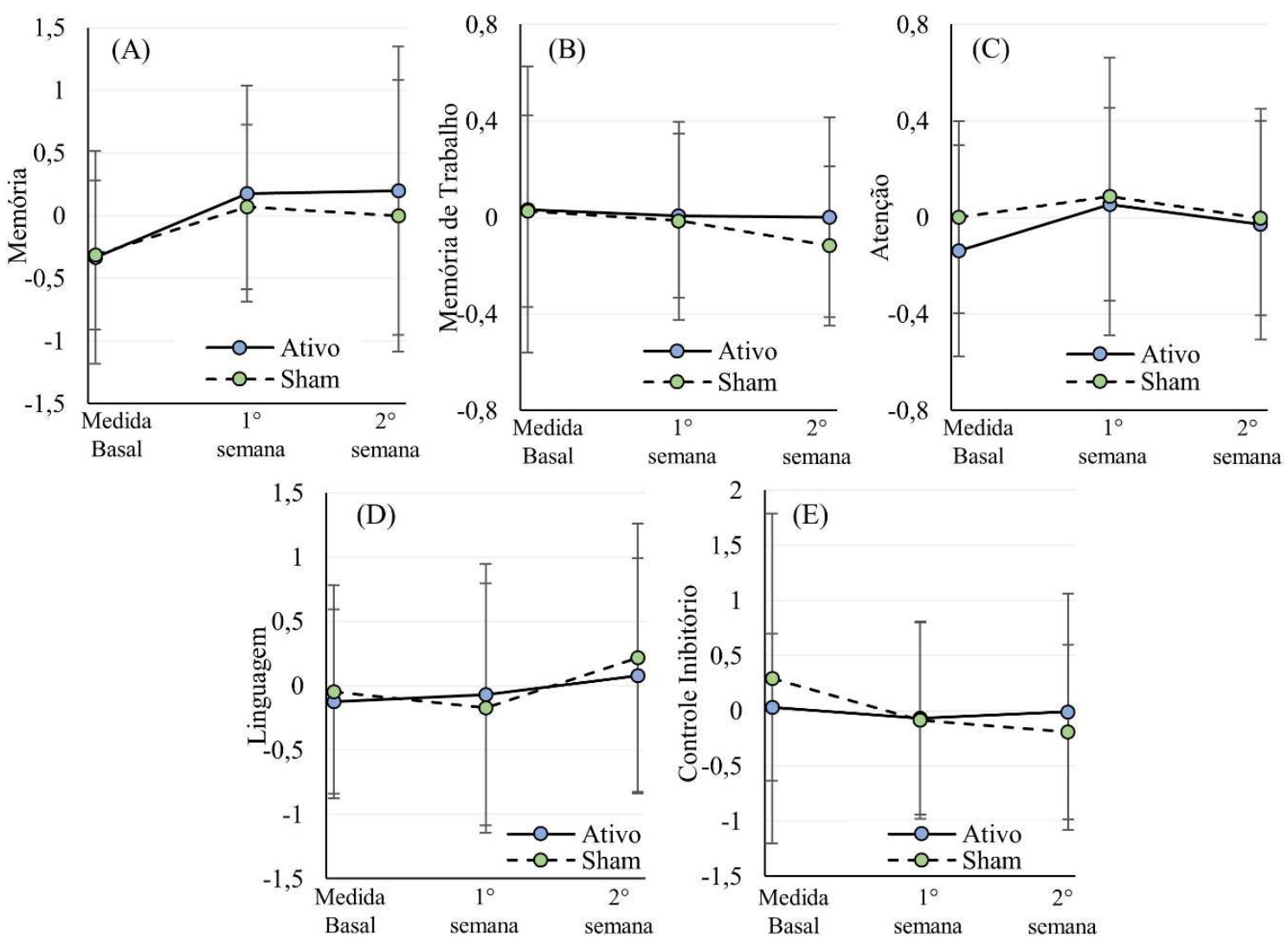

A figura exibe as mudanças cognitivas ao longo do tempo nos grupos ativo e simulado para 5 domínios cognitivos: (a) memória; (b) memória de trabalho; (c) atenção; (d) linguagem; (e) controle inibitório. Barras representam \pm SD. 
Figura 4. Correlação de Pearson entre mudanças em 5 domínios cognitivos: memória; memória de trabalho; atenção; língua; controle inibitório, ao longo do tempo nos grupos ativo (inferior esquerdo) e placebo (lado superior direito).

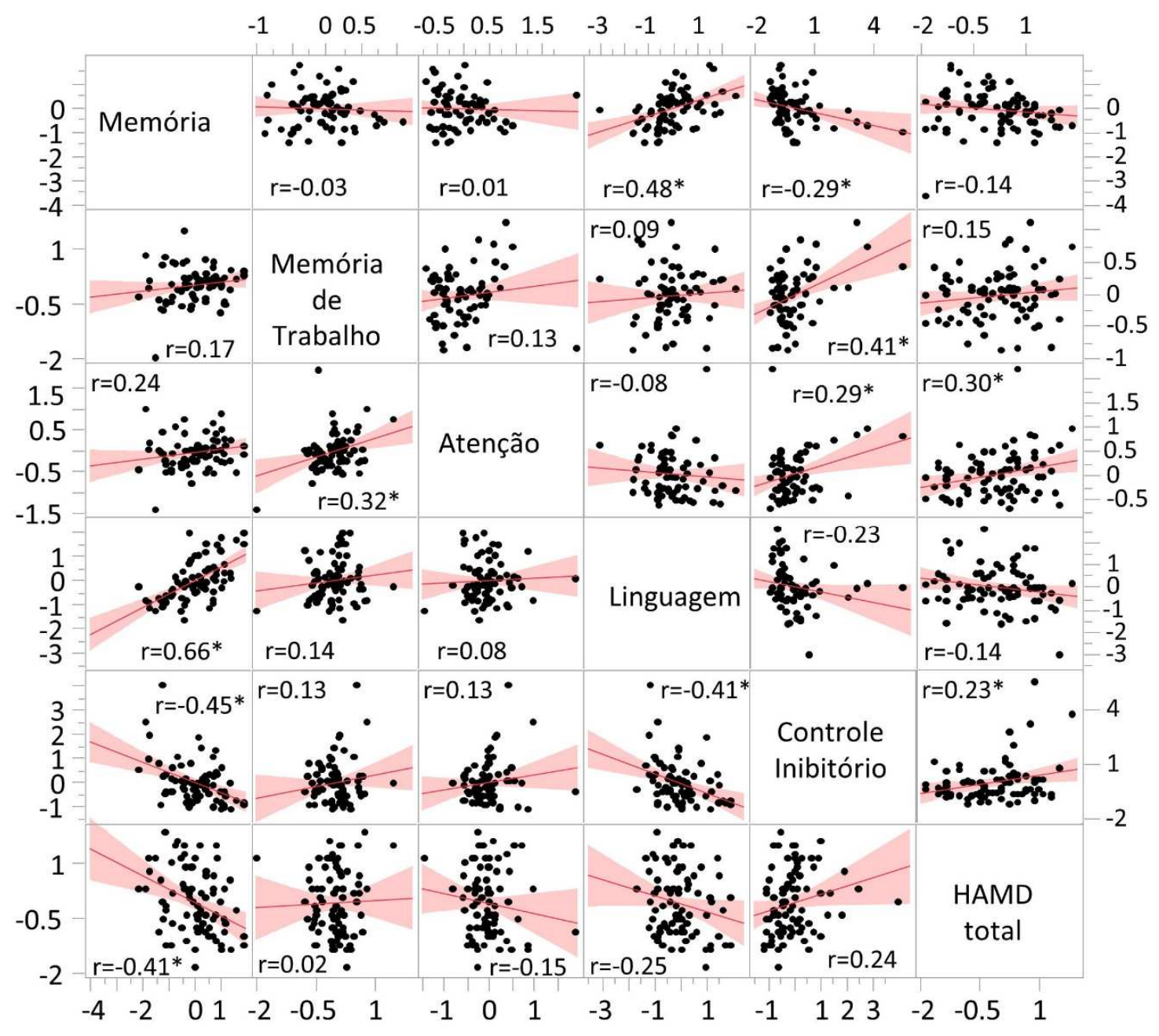

(*) Resultados significativos $(\mathrm{p} \leq 0,05)$

Na Figura 3, acima, observamos no grupo ETCC ativo uma correlação moderada e inversa do domínio memória $(\mathrm{r}=0,41)$ com o escore da melhora da depressão na escala HAMD-17. Para o grupo placebo, houve correlação fraca e direta entre depressão e os domínios de controle inibitório $(r=0,23)$ e atenção $(r=0,30)$. Nenhum declínio cognitivo 
foi observado ao longo do estudo, especialmente no grupo ETCC ativo, indicando a segurança cognitiva da técnica nos parâmetros utilizados em pacientes bipolares com sintomas depressivos. 


\section{DISCUSSÕES}

Sabendo que pacientes com humor deprimido, seja na depressão ou transtorno bipolar apresentam queixa de déficits cognitivos (Millan et al., 2012), este estudo acessou alterações cognitivas em pacientes com depressão bipolar. Não observamos diferenças cognitivas significativas entre os grupos de ETCC e Placebo após 6 semanas de tratamento. Isso sugere que o grupo ativo não apresentou nenhum comprometimento cognitivo da ETCC, de acordo com nossa hipótese. Estes resultados estão de acordo com a literatura pré-existente sobre o uso de ETCC na depressão unipolar, onde a técnica não apresenta uma diminuição na capacidade cognitiva para a população deprimida (Martin et al, 2018). Esse é um achado importante quando comparado às técnicas farmacológicas que podem apresentar efeitos colaterais cognitivos durante o tratamento (Malhi, 2016).

Além da segurança cognitiva da ETCC, a melhora cognitiva da técnica tem sido discutida no campo de pesquisa. Os achados de melhora cognitiva em pacientes deprimidos unipolares são controversos (Tortella et al., 2014; Brunoni et al., 2016a; Martin et al., 2018) e levam a repensar parâmetros da ETCC, como densidade e intensidade de corrente, localização de eletrodos, tempo e frequência de estimulação e atividade de treinamento cognitivo durante a estimulação. Para tanto, sugere-se que sejam realizados estudos clínicos com maior número de sujeitos, utilizando diferentes tipos de montagem e com avaliações cognitivas que possuam diferentes versões dos testes. Não foi observada melhora cognitiva ao longo do tempo para os grupos, sugerindo que a ETCC não promoveu melhora cognitiva após a intervenção. Este achado apoia a sugestão da literatura de que ainda não está claro se o tratamento de ETCC tem benefícios 
cognitivos na depressão (Martin et al., 2018). Como limitação, a bateria de testes utilizados pode não ter sido sensíveis o suficiente para detectar os possíveis efeitos da ETCC na cognição, assim como em outros estudos (Brunoni et al., 2016a) e o número amostral foi inicial estimado para os achados clínicos publicados em Sampaio-Junior (2018). Sugere-se que próximos estudos possam calculem potência amostral especifica para mensurar cognição e possa explorar testes computadorizados, buscando uma maior sensibilidade na medida dos escores.

Neste estudo, não realizamos atividades de estimulação cognitiva em tempo real com pacientes (antes ou durante a aplicação de ETCC). Estes poderiam minimizar os potenciais efeitos da ETCC, uma vez que estudos com pacientes saudáveis (Martin et al., 2014) e deprimidos (Segrave et al., 2014) mostraram melhora maior após a administração de atividades durante e após a estimulação de ETCC, corroborando a ideia que certas tarefas poderiam ativar redes neurais, funcionando como um "alvo funcional" (Jackson et al., 2016), potencializando os efeitos de ETCC. Este estudo apresenta outras limitações, como não comparar um grupo saudável e um grupo de pacientes eutímicos com TB e um número limitado de domínios cognitivos avaliados. Outra limitação para este estudo foi não considerar os possíveis efeitos das diferentes classes de medicações nos modelos de análise cognitivo.

Ainda, outros estudos discutem a identificação de perfis cognitivos heterogêneos no TB, desde um perfil sem comprometimento cognitivo, exceto em crises, podendo apresentar até o comprometimento cognitivo leve iniciado anos antes dos primeiros sintomas, prejuízos cognitivos semelhantes aos dos pacientes esquizofrênicos (Bora et 
al., 2017). Portanto, mais estudos são necessários para analisar os efeitos da ETCC para cada endofenótipo, uma vez que diferenças neurobiológicas podem ser encontradas e levar a mecanismos de ação diferentes. 


\section{CONCLUSÕES}

Assim, mesmo a melhora cognitiva ainda estando sob investigação, a segurança ETCC vem se consolidando no campo psiquiátrico e as técnicas de neuromodulação parecem ser um tratamento alternativo ou complementar, com poucos efeitos colaterais. Embora mais pesquisas sejam necessárias para entender completamente as técnicas de neuromodulação e como potencializá-las, este estudo randomizado, controlado por placebo, duplo-cego, mostrou a segurança cognitiva da ETCC em pacientes com TB. A segurança cognitiva da técnica bem como os possíveis efeitos da melhora cognitiva com ETCC em pacientes bipolares devem continuar a ser investigados em futuras pesquisas explorando o complexo campo dos tratamentos dos transtornos psiquiátricos. 


\section{BIBLIOGRAFIA}

Alghamdi, F., Alhussien, A., Alohali, M., Alatawi, A., Almusned, T., Fecteau, S., ... Bashir, S. (2019). Effect of transcranial direct current stimulation on the number of smoked cigarettes in tobacco smokers. PloS one, 14(2), e0212312. doi:10.1371/journal.pone.0212312

Bora, E., Harrison, B., Yücel, M., \& Pantelis, C. (2013). Cognitive impairment in euthymic major depressive disorder: A meta-analysis. Psychological Medicine, 43(10), 2017-2026. doi:10.1017/S0033291712002085

Brunoni, A. R., Amadera, J., Berbel, B., Volz, M. S., Rizzerio, B. G., \& Fregni, F. (2011). A systematic review on reporting and assessment of adverse effects associated with transcranial direct current stimulation. International Journal of Neuropsychopharmacology, 14(8), 1133-1145. doi:10.1017/s1461145710001690

Brunoni AR, Moffa AH, Fregni F, Palm U, Padberg F, Blumberger DM, Daskalakis ZJ, Bennabi D, Haffen E, Alonzo A, Loo CK. (2016). Transcranial direct current stimulation for acute major depressive episodes: meta-analysis of individual patient data. The British journal of psychiatry : the journal of mental science,208(6), 522-531. doi:10.1192/bjp.bp.115.164715

Brunoni, A. R., Valiengo, L., Baccaro, A., Zanão, T. A., De Oliveira, J. F., Goulart, A., ... Fregni, F. (2013). The Sertraline vs Electrical Current Therapy for Treating Depression Clinical Study. JAMA Psychiatry, 70(4), 383. doi:10.1001/2013.jamapsychiatry.32

Brunoni, A. R., Tortella, G., Benseñor, I. M., Lotufo, P. A., Carvalho, A. F., \& Fregni, F. (2016a). Cognitive effects of transcranial direct current stimulation in depression: Results from the SELECT-TDCS trial and insights for further clinical trials. Journal of Affective Disorders, 202, 46-52. doi:10.1016/j.jad.2016.03.066 
Brunoni, Andre R., Sampaio-Junior, Bernardo, Moffa, Adriano H., Aparício, Luana V., Gordon, Pedro, Klein, Izio, Rios, Rosa M., Razza, Lais B., Loo, Colleen, Padberg, Frank, \& Valiengo, Leandro. (2019). Noninvasive brain stimulation in psychiatric disorders: a primer. Brazilian Journal of Psychiatry,41(1), 70-81. Epub October 11, 2018.https://dx.doi.org/10.1590/1516-4446-2017-0018

Martin, D. M., Moffa, A., Nikolin, S., Bennabi, D., Brunoni, A. R., Flannery, W., ... Loo, C. K. (2018). Cognitive effects of transcranial direct current stimulation treatment in patients with major depressive disorder: An individual patient data meta-analysis of randomised, sham-controlled trials. Neuroscience \& Biobehavioral Reviews, 90, 137-145. doi:10.1016/j.neubiorev.2018.04.008

Brunoni, A. R., $\quad$ Moffa, A. H., $\quad$ Sampaio-Junior, B., $\quad$ Borrione, L., $\quad$ Moreno, M. L., Fernandes, R. A., ... Benseñor, I. M. (2017). Trial of Electrical Direct-Current Therapy versus Escitalopram for Depression. New England Journal of Medicine, 376(26), 25232533. doi:10.1056/nejmoa1612999

Depp, C. A., Mausbach, B. T., Harmell, A. L., Savla, G. N., Bowie, C. R., Harvey, P. D., \& Patterson, T. L. (2012). Meta-analysis of the association between cognitive abilities and everyday functioning in bipolar disorder. Bipolar Disorders, 14(3), 217-226. doi:10.1111/j.1399-5618.2012.01011.x

Dondé, C., Neufeld, N. H., \& Geoffroy, P. A. (2018). The Impact of Transcranial Direct Current Stimulation (tDCS) on Bipolar Depression, Mania, and Euthymia: a Systematic Review of Preliminary Data. Psychiatric Quarterly, 89(4), 855-867. doi:10.1007/s11126018-9584-5

Duarte, D., Castelo-Branco, L. E., Uygur Kucukseymen, E., \& Fregni, F. (2018). Developing an optimized strategy with transcranial direct current stimulation to enhance 
the endogenous pain control system in fibromyalgia. Expert Review of Medical Devices, 15(12), 863-873. doi:10.1080/17434440.2018.1551129

Fregni, F., \& Pascual-Leone, A. (2007). Technology Insight: noninvasive brain stimulation in neurology_perspectives on the therapeutic potential of rTMS and tDCS. Nature Clinical Practice Neurology, 3(7), 383-393. doi:10.1038/ncpneuro0530

Geddes, J. R., \& Miklowitz, D. J. (2013). Treatment of bipolar disorder. The Lancet, 381(9878), 1672-1682. doi:10.1016/s0140-6736(13)60857-0

Gigante, A. D., $\quad$ Young, L. T., $\quad$ Yatham, L. N., $\quad$ Andreazza, A. C., $\quad$ Nery, F. G., Grinberg, L. T., ... Lafer, B. (2010). Morphometric post-mortem studies in bipolar disorder: possible association with oxidative stress and apoptosis. The International Journal of Neuropsychopharmacology, 14(08), 1075-1089. doi:10.1017/s146114571000146x

Goodwin, F. K., \& Jamison, K. R. (2007). Manic-Depressive Illness: Bipolar Disorders and Recurrent Depression. New York, NY: Oxford University Press.

Grunze, H., Vieta, E., Goodwin, G. M., Bowden, C., Licht, R. W., \& Möller, H. (2013). The World Federation of Societies of Biological Psychiatry (WFSBP) Guidelines for the Biological Treatment of Bipolar Disorders: Update 2012 on the long-term treatment of bipolar disorder. The World Journal of Biological Psychiatry, 14(3), 154-219. doi:10.3109/15622975.2013.770551

Harvey, P. D., $\quad$ Hassman, H., $\quad$ Mao, L., $\quad$ Gharabawi, G. M., Mahmoud, R. A., ～\& Engelhart, L. M. (2007). Cognitive Functioning and Acute Sedative Effects of Risperidone and Quetiapine in Patients With Stable Bipolar I Disorder. The Journal of Clinical Psychiatry, 68(08), 1186-1194. doi:10.4088/jcp.v68n0804 
Jackson, M. P., Rahman, A., Lafon, B., Kronberg, G., Ling, D., Parra, L. C., \& Bikson, M. (2016). Animal models of transcranial direct current stimulation: Methods and mechanisms. Clinical neurophysiology : official journal of the International Federation of Clinical Neurophysiology, 127(11), 3425-3454. doi:10.1016/j.clinph.2016.08.016

Kalu, U., Sexton, C., Loo, C., \& Ebmeier, K. (2012). Transcranial direct current stimulation in the treatment of major depression: A meta-analysis. Psychological Medicine, 42(9), 1791-1800. doi:10.1017/S0033291711003059

Kent, P. (2013). The Evolution of the Wechsler Memory Scale: A Selective Review. Applied Neuropsychology: Adult, 20(4), 277-291. doi:10.1080/09084282.2012.689267

Lima, F., Rabelo-da-Ponte, F. D., Bücker, J., C Czepielewski, L., Hasse-Sousa, M., Telesca, R., ... Rosa, A. R. (2019). Identifying cognitive subgroups in bipolar disorder: A cluster analysis. Journal of Affective Disorders, 246, 252-261. doi:10.1016/j.jad.2018.12.044

Liu, C. S., Rau, A., Gallagher, D., Rajji, T. K., Lanctôt, K. L., \& Herrmann, N. (2017). Using transcranial direct current stimulation to treat symptoms in mild cognitive impairment and Alzheimer's disease. Neurodegenerative Disease Management, 7(5), 317329. doi:10.2217/nmt-2017-0021

Lolas F. Brain polarization: behavioral and therapeutic effects. Biol Psychiatry. 1977 Feb;12(1):37-47.

Loo, C. K., Alonzo, A., Martin, D., Mitchell, P. B., Galvez, V., \& Sachdev, P. (2012). Transcranial direct current stimulation for depression: 3-week, randomised, shamcontrolled trial. British Journal of Psychiatry, 200(1), 52-59. doi:10.1192/bjp.bp.111.097634 
Loo, C. K., Husain, M. M., McDonald, W. M., Aaronson, S., O'Reardon, J. P., Alonzo, A., ... McClintock, S. M. (2018). International randomized-controlled trial of transcranial Direct Current Stimulation in depression. Brain Stimulation, 11(1), 125-133. doi:10.1016/j.brs.2017.10.011

Magalhães, S. S., \& Hamdan, A. C. (2010). The Rey Auditory Verbal Learning Test: Normative data for the Brazilian population and analysis of the influence of demographic variables. Psychology \& Neuroscience, 3(1), 85-91. doi:10.3922/j.psns.2010.1.011

Malhi, G. S., McAulay, C., Gershon, S., Gessler, D., Fritz, K., Das, P., \& Outhred, T. (2016). The Lithium Battery: assessing the neurocognitive profile of lithium in bipolar disorder. Bipolar Disorders, 18(2), 102-115. doi:10.1111/bdi.12375

Martin, D. M., Moffa, A., Nikolin, S., Bennabi, D., Brunoni, A. R., Flannery, W., ... Loo, C. K. (2018). Cognitive effects of transcranial direct current stimulation treatment in patients with major depressive disorder: An individual patient data meta-analysis of randomised, sham-controlled trials. Neuroscience \& Biobehavioral Reviews, 90, 137-145. doi:10.1016/j.neubiorev.2018.04.008

Martin, D. M., Liu, R., Alonzo, A., Green, M., \& Loo, C. K. (2014). Use of transcranial direct current stimulation (tDCS) to enhance cognitive training: effect of timing of stimulation. Experimental Brain Research, 232(10), 3345-3351. doi:10.1007/s00221-0144022-x

Merikangas, K. R., Jin, R., He, J., Kessler, R. C., Lee, S., Sampson, N. A., ... Zarkov, Z. (2011). Prevalence and Correlates of Bipolar Spectrum Disorder in the World Mental Health Survey Initiative. Archives of General Psychiatry, 68(3), 241. doi:10.1001/archgenpsychiatry.2011.12 
Millan, M. J., Agid, Y., Brüne, M., Bullmore, E. T., Carter, C. S., Clayton, N. S., .. Young, L. J. (2012). Cognitive dysfunction in psychiatric disorders: characteristics, causes and the quest for improved therapy. Nature Reviews Drug Discovery, 11(2), 141-168. doi: $10.1038 / \operatorname{nrd} 3628$

Miranda, P. C., Lomarev, M., \& Hallett, M. (2006). Modeling the current distribution during transcranial direct current stimulation. Clinical Neurophysiology, 117(7), 16231629. doi:10.1016/j.clinph.2006.04.009

Mutz, J., Edgcumbe, D. R., Brunoni, A. R., \& Fu, C. H. (2018). Efficacy and acceptability of non-invasive brain stimulation for the treatment of adult unipolar and bipolar depression: A systematic review and meta-analysis of randomised sham-controlled trials. Neuroscience \& Biobehavioral Reviews, 92, 291-303. doi:10.1016/j.neubiorev.2018.05.015

Myczkowski, M. L., Fernandes, A., Moreno, M., Valiengo, L., Lafer, B., Moreno, R. A., ... Brunoni, A. R. (2018). Cognitive outcomes of TMS treatment in bipolar depression: Safety data from a randomized controlled trial. Journal of Affective Disorders, 235, 20-26. doi:10.1016/j.jad.2018.04.022

Nitsche, M. A., Liebetanz, D., Antal, A., Lang, N., Tergau, F., \& Paulus, W. (2003). Chapter 27 Modulation of cortical excitability by weak direct current stimulation technical, safety and functional aspects. Transcranial Magnetic Stimulation and Transcranial Direct Current Stimulation, Proceedings of the 2nd International Transcranial Magnetic Stimulation (TMS) and Transcranial Direct Current Stimulation (tDCS) Symposium, 255-276. doi:10.1016/s1567-424x(09)70230-2

Nitsche, M. A., \& Paulus, W. (2000). Excitability changes induced in the human motor cortex by weak transcranial direct current stimulation. The Journal of Physiology, 527(3), 633-639. doi:10.1111/j.1469-7793.2000.t01-1-00633.x 
Pereira Junior, B. D., Tortella, G., Lafer, B., Nunes, P., Benseñor, I. M., Lotufo, P. A., ... Brunoni, A. R. (2015). The Bipolar Depression Electrical Treatment Trial (BETTER): Design, Rationale, and Objectives of a Randomized, Sham-Controlled Trial and Data from the Pilot Study Phase. Neural Plasticity, 2015, 1-10. doi:10.1155/2015/684025

Perret, E. (1974). The left frontal lobe of man and the suppression of habitual responses in verbal categorical behaviourt. Neuropsychologia, 12(3), 323-330. doi:10.1016/00283932(74)90047-5

Phillips, M. L., \& Kupfer, D. J. (2013). Bipolar disorder diagnosis: challenges and future directions. The Lancet, 381(9878), 1663-1671. doi:10.1016/s0140-6736(13)60989-7

Rachid, F. (2016). Neurostimulation techniques in the treatment of nicotine dependence: A review. The American Journal on Addictions, 25(6), 436-451. doi:10.1111/ajad.12405

Reitan, R. M. (1958). Validity of the Trail Making Test as an Indicator of Organic Brain Damage. Perceptual and Motor Skills, 8(3), 271-276. doi:10.2466/pms.1958.8.3.271

Roizenblatt, S., Fregni, F., Gimenez, R., Wetzel, T., Rigonatti, S. P., Tufik, S., . . Valle, A. C. (2007). Site-specific Effects of Transcranial Direct Current Stimulation on Sleep and Pain in Fibromyalgia: A Randomized, Sham-controlled Study. Pain Practice, 7(4), 297-306. doi:10.1111/j.1533-2500.2007.00152.x

Sampaio-Junior, B., $\quad$ Tortella, G., $\quad$ Borrione, L., $\quad$ Moffa, A. H., $\quad$ Machado-Vieira, R., Cretaz, E., ... Brunoni, A. R. (2018). Efficacy and Safety of Transcranial Direct Current Stimulation as an Add-on Treatment for Bipolar Depression. JAMA Psychiatry, 75(2), 158. doi:10.1001/jamapsychiatry.2017.4040

Schulze, L., Wheeler, S., McAndrews, M. P., Solomon, C. J., Giacobbe, P., \& Downar, J. (2016). Cognitive safety of dorsomedial prefrontal repetitive transcranial magnetic 
stimulation in major depression. European Neuropsychopharmacology, 26(7), 1213-1226. doi:10.1016/j.euroneuro.2016.04.004

Segrave, R., Arnold, S., Hoy, K., \& Fitzgerald, P. (2014). Concurrent Cognitive Control Training Augments the Antidepressant Efficacy of tDCS: A Pilot Study. Brain Stimulation, 7(2), 325-331. doi:10.1016/j.brs.2013.12.008

Seibt, O., Brunoni, A. R., Huang, Y., \& Bikson, M. (2015). The Pursuit of DLPFC: Nonneuronavigated Methods to Target the Left Dorsolateral Pre-frontal Cortex With Symmetric Bicephalic Transcranial Direct Current Stimulation (tDCS). Brain Stimulation, 8(3), 590602. doi:10.1016/j.brs.2015.01.401

Sienaert, P., Lambrichts, L., Dols, A., \& De Fruyt, J. (2012). Evidence-based treatment strategies for treatment-resistant bipolar depression: a systematic review. Bipolar Disorders, 15(1), 61-69. doi:10.1111/bdi.12026

Spreen, O., \& Strauss, E. (1998). A Compendium of Neuropsychological Tests: Administration, Norms, and Commentary. New York, NY: Oxford University Press.

Tortella, G., Casati, R., Aparicio, L. V., Mantovani, A., Senço, N., D'Urso, G., ... Brunoni, A. R. (2015). Transcranial direct current stimulation in psychiatric disorders. World journal of psychiatry, 5(1), 88-102. doi:10.5498/wjp.v5.i1.88

Tortella, G., Selingardi, P., Moreno, M., Veronezi, B., \& Brunoni, A. (2015). Does NonInvasive Brain Stimulation Improve Cognition in Major Depressive Disorder? A Systematic Review. CNS \& Neurological Disorders - Drug Targets, 13(10), 1759-1769. doi:10.2174/1871527313666141130224431

Vacas, S. M., Stella, F., Loureiro, J. C., Simões do Couto, F., Oliveira-Maia, A. J., \& Forlenza, O. V. (2018). Noninvasive brain stimulation for behavioural and psychological 
symptoms of dementia: A systematic review and meta-analysis. International Journal of Geriatric Psychiatry, 34(9), 1336-1345. doi:10.1002/gps.5003

Yatham, L. N., Kennedy, S. H., Parikh, S. V., Schaffer, A., Beaulieu, S., Alda, M., ... Berk, M. (2012). Canadian Network for Mood and Anxiety Treatments (CANMAT) and International Society for Bipolar Disorders (ISBD) collaborative update of CANMAT guidelines for the management of patients with bipolar disorder: update 2013. Bipolar Disorders, 15(1), 1-44. doi:10.1111/bdi.12025

Yatham, L. N., Torres, I. J., Malhi, G. S., Frangou, S., Glahn, D. C., Bearden, C. E., ... Chengappa, K. N. (2010). The International Society for Bipolar Disorders-Battery for Assessment of Neurocognition (ISBD-BANC). Bipolar Disorders, 12(4), 351-363. doi:10.1111/j.1399-5618.2010.00830.x 